\title{
Bethe Ansatz and exact form factors of the $O(N)$ Gross Neveu-model
}

\author{
Hrachya M. Babujian, ${ }^{a, b}$ Angela Foerster ${ }^{c}$ and Michael Karowski ${ }^{d}$ \\ ${ }^{a}$ Yerevan Physics Institute, \\ Alikhanian Brothers 2, Yerevan, 375036 Armenia \\ ${ }^{b}$ International Institute of Physics, Universidade Federal do Rio Grande do Norte (UFRN), \\ 59078-400 Natal-RN, Brazil \\ ${ }^{c}$ Instituto de Física da UFRGS, \\ Av. Bento Gonçalves 9500, Porto Alegre, RS, Brazil \\ ${ }^{d}$ Institut für Theoretische Physik, FU-Berlin, \\ Arnimallee 14, 14195 Berlin, Germany \\ E-mail: babujian@yerphi.am, angela@if.ufrgs.br, \\ karowski@physik.fu-berlin.de
}

ABStract: We apply previous results on the $O(N)$ Bethe Ansatz [1-3] to construct a general form factor formula for the $O(N)$ Gross-Neveu model. We examine this formula for several operators, such as the energy momentum, the spin-field and the current. We also compare these results with the $1 / N$ expansion of this model and obtain full agreement. We discuss bound state form factors, in particular for the three particle form factor of the field. In addition for the two particle case we prove a recursion relation for the K-functions of the higher level Bethe Ansatz.

KEYwords: Field Theories in Lower Dimensions, Bethe Ansatz, Integrable Field Theories ARXIV EPRINT: 1510.08784 


\section{Contents}

1 Introduction 1

2 General settings $\quad 4$

2.1 The $O(N)$-Gross-Neveu S-matrix 4

$\begin{array}{lll}2.1 .1 & \text { Bound states }\end{array}$

3 Generalized form factors $\quad 6$

$4 O(N)$ form factors and Bethe Ansatz $\quad 8$

4.1 The fundamental theorem 8

$\begin{array}{lr}4.2 \text { Higher level off-shell Bethe Ansatz } & 10\end{array}$

5 Examples $\quad 12$

$\begin{array}{lll}5.1 \text { Current } & 12\end{array}$

$\begin{array}{lll}5.2 & \text { Field } & 13\end{array}$

$\begin{array}{lll}5.3 & \text { Energy momentum } & 15\end{array}$

6 Conclusions 16

$\begin{array}{ll}\text { A Proof of the main theorem } 1 & 17\end{array}$

$\begin{array}{ll}\text { B Two-particle current form factor } & 19\end{array}$

C Higher level K-functions $\quad 20$

$\begin{array}{lll}\text { C.1 Proof of lemma } 2 & 20\end{array}$

$\begin{array}{lll}\text { C.2 Two-particle higher level K-functions } & 21\end{array}$

D Bound state form factors $\quad 24$

$\begin{array}{lll}\text { E } & 1 / \mathrm{N} \text { expansion } & \mathbf{2 7}\end{array}$

E.1 1/N expansion of the exact 3-particle field form factor 27

E.2 $1 / \mathrm{N}$ perturbation theory 28

\section{Introduction}

The $O(N) \sigma$ - and Gross-Neveu (GN) models are integrable and asymptotically free quantum field theories in $1+1$ dimension. The S-matrices of these two models correspond to two solutions of the Yang-Baxter equation $[4,5]$. In previous articles we constructed the $O(N)$ nested off-shell Bethe Ansatz [1,2] and applied this technique to construct the exact form factors for the $O(N) \sigma$ model [3]. Here we extend this work and construct the form factors 
for the $O(N)$ Gross-Neveu model for arbitrary number of fundamental particles (for the two-particle case see [6]). The model exhibits a very rich bound state structure and kinks (see e.g. [7]), turning this study even more challenging.

Before we recall the S-matrix and all other details of this model we should mention that the integrable structure present in $1+1$ dimension is now becoming relevant and actual in higher dimensional gauge theories under specific circumstances. Remarkably, in the articles [8-11] (see also references therein) a non-perturbative formulation of planar scattering in the $N=4$ Supersymmetric Yang-Mills theory (SYM) with the so called polygonal Wilson loops was proposed and a new decomposition of the Wilson loops in terms of the fundamental building blocks-Pentagon transitions was introduced. These transitions are directly related to the dynamics of the Gubser-Klebanov-Polyakov flux-tube [12], which can be computed exactly by exploring the integrability. In addition, three axioms about the transitions that single particles must satisfy were postulated and, interestingly, it is possible to verify that these axioms correspond to some deformations of the form factor equations in $1+1$ - dimensional integrable quantum field theories. Such exact and constructive developments in the $N=4 \mathrm{SYM}$ theory opens, indeed, large perspectives in the view of using the exact integrability and the full machinery of the form factor program to get physical insights, specially in the case of non-trivial symmetry groups, such as $\mathrm{SU}(N)$ and $O(N)$.

In this article we consider the $O(N)$-Gross-Neveu model for $N=$ even. We do not use any Lagrangian to construct the model, nevertheless, we give the following motivation. The $O(N)$-Gross-Neveu model describes the interaction of $N / 2$ Dirac (or $N$ Majorana) fermions defined by the Lagrangian ${ }^{1}[13]$

$$
\mathcal{L}^{\mathrm{GN}}=\sum_{\alpha=1}^{N / 2} \bar{\psi}_{\alpha} i \gamma \partial \psi_{\alpha}+\frac{1}{2} g^{2}\left(\sum_{i=1}^{N / 2} \bar{\psi}_{\alpha} \psi_{\alpha}\right)^{2} .
$$

It is known from semi-classical calculations [14] that there are bound states of two fundamental fermions in the scalar and the anti-symmetric tensor channel. Furthermore there are kinks such that the fundamental fermions are kink-kink bound states. The bootstrap program does not use the Lagrangian, but we are looking for an factorizing S-matrix of an $O(N)$-isovector $N$-plett of self conjugate fundamental fermions. However, now we assume bound states in the scalar and anti-symmetric tensor channel of two of them.

In this article we use the techniques of $[1,3]$ to construct the form factors of the $O(N)$-Gross-Neveu model. We apply the general results to compute exact form factors for the energy-momentum, the spin-field and the current. The exact results are compared with the ones obtained in perturbation theory using the $1 / N$ expansion. The final aim of the form factor program is to obtain explicit results for the correlation functions or Wightman functions in the framework of 2-dimensional integrable QFTs. In $[6,15]$ the concept of generalized form factors was introduced and developed further by Smirnov [16]. We call the matrix elements of fields with many particle states: "generalized form factors".

\footnotetext{
${ }^{1}$ The Lagrangian (1.1) is invariant under $O(N)$ transformations of the vector of $N$ Majorana fermi fields $\psi_{\alpha}^{(i)}(\alpha=1, \ldots, N / 2), i=1,2$, where $\psi_{\alpha}=\psi_{\alpha}^{(1)}+i \psi_{\alpha}^{(2)}[5]$.
} 
Matrix difference equations (the generalized Watson's equations) are solved by using the "off-shell Bethe Ansatz" [1, 17-19], which was introduced in [20] to solve the KnizhnikZamolodchikov equations. Other approaches to form factors in integrable quantum field theories can be found in [21-29]. For articles considering the form factor program for Bethe Ansatz solvable models with nesting see also [30-33].

The general form factor formula in terms of an integral representation is the main result of this paper. It solves the form factors equations. The matrix element of a local operator $\mathcal{O}(x)$ for a state of $n$ particles of kind $\alpha_{i}$ with rapidities $\theta_{i}$

$$
\left\langle 0|\mathcal{O}(x)| \theta_{1}, \ldots, \theta_{n}\right\rangle_{\underline{\alpha}}^{\text {in }}=e^{-i x\left(p_{1}+\cdots+p_{n}\right)} F_{\underline{\alpha}}^{\mathcal{O}}(\underline{\theta})
$$

defines the generalized form factor $F_{\alpha}^{\mathcal{O}}(\underline{\theta})$. Here we restrict $\alpha$ to the fundamental particles of the model, which form an isovector $N$-plett of $O(N)$. Following [6] we write

$$
F_{\underline{\alpha}}^{\mathcal{O}}(\underline{\theta})=K_{\underline{\alpha}}^{\mathcal{O}}(\underline{\theta}) \prod_{1 \leq i<j \leq n} F\left(\theta_{i j}\right)
$$

where $F(\theta)$ is the minimal form factor function.

For the K-function we propose the same Ansatz as for the $\sigma$-model in [3] in terms of a nested 'off-shell' Bethe Ansatz

$$
K_{\underline{\alpha}}^{\mathcal{O}}(\underline{\theta})=N_{n}^{\mathcal{O}} \int_{\mathcal{C}_{\underline{\theta}}^{(1)}} d z_{1} \cdots \int_{\mathcal{C}_{\underline{\theta}}^{(m)}} d z_{m} \tilde{h}(\underline{\theta}, \underline{z}) p^{\mathcal{O}}(\underline{\theta}, \underline{z}) \tilde{\Psi}_{\underline{\alpha}}(\underline{\theta}, \underline{z}) .
$$

Here $\tilde{h}(\underline{\theta}, \underline{z})$ is a scalar function which depends only on the S-matrix. The scalar p-function $p^{\mathcal{O}}(\underline{\theta}, \underline{z})$ which is in general a simple function of $e^{\theta_{i}}$ and $e^{z_{j}}$ depends on the specific operator $\mathcal{O}(x)$. This Ansatz transforms the complicated form factor matrix equations (see (3.1)-(3.3) below) to simple equations for the scalar function $p^{\mathcal{O}}(\underline{\theta}, \underline{z})$ (see also [19]). The integration contour $\mathcal{C}_{\underline{\theta}}$ will be specified in section 4 . The state $\tilde{\Psi}_{\underline{\alpha}}$ in (1.4) is a linear combination of the basic Bethe Ansatz co-vectors (see [3] and (4.1))

$$
\tilde{\Psi}_{\underline{\alpha}}(\underline{\theta}, \underline{z})=L_{\underline{\beta}}(\underline{z}) \tilde{\Phi}_{\underline{\alpha}}^{\stackrel{\leftrightarrow}{\beta}}(\underline{\theta}, \underline{z}) .
$$

The nested off-shell Bethe Ansatz is obtained by making for $L_{\hat{\beta}}(\underline{z})$ an Ansatz like (1.4) and iterating this procedure. In the present paper we mainly consider the case where $\alpha$ correspond to the fundamental fermions of the $O(N)$-Gross-Neveu model Lagrangian (1.1). In forthcoming publications we will consider the kinks [34] and we will discuss, in particular, the $O(6)$-Gross-Neveu model in more detail [35].

The 'off-shell' Bethe Ansatz states are highest weight states if they satisfy certain matrix difference equations (see for instance [1]). For $n$ particle states the $O(N)$ weights are

$$
\left(w_{1}, \ldots, w_{N / 2}\right)=\left(n-n_{1}, \ldots, n_{N / 2-2}-n_{-}-n_{+}, n_{-}-n_{+}\right)
$$

where $n_{1}=m, n_{2}, \ldots$ are the numbers of integrations in (1.4) and the higher levels of the nesting. In particular $n_{ \pm}$are the numbers of positive/negative chirality spinors. The various levels of the nested Bethe Ansatz correspond to the nodes of the Dynkin diagram 
of the corresponding Lie algebra (see for instance [36-38] and references therein). Here we have $D_{N / 2}$ for $N=$ even (see figure 2). In [3] we used for the $O(N) \sigma$-model the group isomorphy $O(4) \simeq \mathrm{SU}(2) \otimes \mathrm{SU}(2)$ to start the nesting procedure with form factors of the $\mathrm{SU}(2)$ chiral Gross-Neveu model [39]. For the $O(N)$ Gross-Neveu model it is also possible to use the group isomorphy $O(6) \simeq \mathrm{SU}(4)$ to start the nesting with form factors of the SU(4) chiral Gross-Neveu model [39]. This will be performed in detail in a separate paper [35]. For the on-shell Bethe Ansatz for $N$ even see also [40].

Section 2 provides some known results and the notation for the $O(N)$ Gross-Neveu S-matrix, the bound states, etc. In section 3 we recall the general form factor equations and obtain the minimal form factor function. In section 4 we present the general exact form factors formula for the $O(N)$-Gross-Neveu model and discuss the higher levels of the nested off-shell Bethe Ansatz. In section 5 the general results are applied to some examples. The more complicated proofs and calculations are delegated to the appendices.

\section{General settings}

\subsection{The $O(N)$-Gross-Neveu S-matrix}

We consider the fundamental particles of the Lagrangian (1.1) which are fermions and transform as the vector representation of $O(N)$. The structure of the S-matrix is the same as that of the nonlinear $\sigma$-model [3] however, here we are looking for a solution of the $O(N)$-Yang-Baxter equations with a bound state pole in the physical strip $0<$ $\operatorname{Im} \theta<\pi$. Therefore, here "minimality" implies that the S-matrix for the scattering of two fundamental particles is of the form

$$
S(\theta, N)=\frac{\sinh \theta+i \sin \pi \nu}{\sinh \theta-i \sin \pi \nu} S^{\min }(\theta), \quad \text { with } \nu=\frac{2}{N-2} .
$$

This S-matrix was given by Zamolodchikov-Zamolodchikov [5]. The first factor in (2.1) is the sine-Gordon breather-breather [41] amplitude and $S^{\text {min }}$ is the minimal $O(N)$ S-matrix which is the one of the nonlinear $\sigma$-model (see e.g. [3]). The position of the pole is dictated by the condition [42] that the pole has to be cancelled by a zero in the amplitude $S_{+}^{\mathrm{min}}$. This condition ${ }^{2}$ fixes the pole and therefore the bound state mass spectrum

$$
m_{k}=2 m \sin \frac{1}{2} k \pi \nu \quad(k=1,2, \ldots, N / 2-2) .
$$

For each "principal" quantum number $k$ there exist particles $b_{k}^{(r)}$ which are anti-symmetric tensors of rank $r=k, k-2, \cdots \geq 0$, i.e. they transform according to the $r$-th fundamental representation of $O(N)$. These particles are bosons/fermions for $k$ even/odd. In addition there exist "kinks" of mass $m$ which transform as the two spinor representations of $O(N)$ (with positive or negative isotopic chirality).

Note the intimate connection between the spectrum of the GN-model, figure 1, and the Dynkin diagram figure 2. There exist exclusively such one-particle states which transform according to one of the fundamental (or trivial) representations of $O(N)$.

\footnotetext{
${ }^{2}$ An additional pole in $S_{+}^{\mathrm{GN}}$ would contradict positivity in the Hilbert space (for details see [42]).
} 


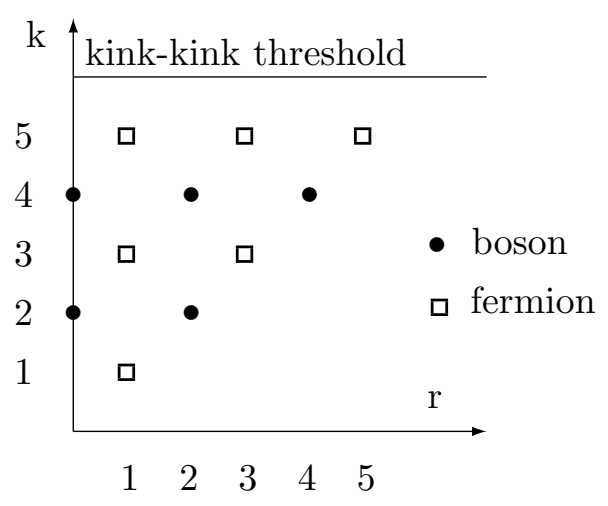

Figure 1. Particle spectrum of the $O(N)$-Gross-Neveu model for $N=14$.

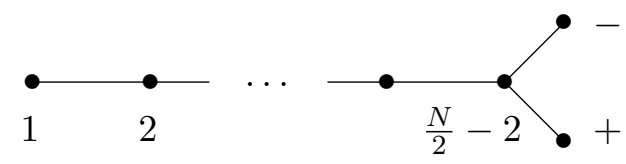

Figure 2. Dynkin diagram for $O(N)$.

For the Bethe Ansatz it is convenient as in [3] to use instead of the real basis $|\alpha\rangle_{r},(\alpha=1,2, \ldots, N)$ the complex basis $|1\rangle,|2\rangle, \ldots,|\overline{2}\rangle,|\overline{1}\rangle$

$$
\left.\begin{array}{l}
|\alpha\rangle=\frac{1}{\sqrt{2}}\left(|2 \alpha-1\rangle_{r}+i|2 \alpha\rangle_{r}\right) \\
|\bar{\alpha}\rangle=\frac{1}{\sqrt{2}}\left(|2 \alpha-1\rangle_{r}-i|2 \alpha\rangle_{r}\right)
\end{array}\right\}, \quad \alpha=1,2, \ldots, N / 2 .
$$

Then the S-matrix writes in terms of the components as

$$
S_{\alpha \beta}^{\delta \gamma}(\theta)=b(\theta) \delta_{\alpha}^{\gamma} \delta_{\beta}^{\delta}+c(\theta) \delta_{\alpha}^{\delta} \delta_{\beta}^{\gamma}+d(\theta) \mathbf{C}^{\delta \gamma} \mathbf{C}_{\alpha \beta}
$$

with the rapidity difference $\theta$ of the particles and the "charge conjugation matrices"

$$
\mathbf{C}_{\alpha \beta}=\delta_{\alpha \bar{\beta}} \text { and } \mathbf{C}^{\alpha \beta}=\delta^{\alpha \bar{\beta}} .
$$

The Yang-Baxter-, crossing- and unitarity-relation write as in [3]. The highest weight amplitude is $a(\theta)=S_{+}(\theta)=b(\theta)+c(\theta)$

$$
\begin{aligned}
a(\theta) & =\exp \left(2 \int_{0}^{\infty} \frac{d t}{t}\left(\frac{e^{-t(1-\nu)}-e^{-t}}{1+e^{-t}}\right) \sinh t \frac{\theta}{i \pi}\right) \\
& =\frac{\Gamma\left(1-\frac{1}{2 \pi i} \theta\right) \Gamma\left(\frac{1}{2}+\frac{1}{2 \pi i} \theta\right)}{\Gamma\left(1+\frac{1}{2 \pi i} \theta\right) \Gamma\left(\frac{1}{2}-\frac{1}{2 \pi i} \theta\right)} \frac{\Gamma\left(1-\frac{1}{2} \nu+\frac{1}{2 \pi i} \theta\right) \Gamma\left(\frac{1}{2}-\frac{1}{2} \nu-\frac{1}{2 \pi i} \theta\right)}{\Gamma\left(1-\frac{1}{2} \nu-\frac{1}{2 \pi i} \theta\right) \Gamma\left(\frac{1}{2}-\frac{1}{2} \nu+\frac{1}{2 \pi i} \theta\right)}
\end{aligned}
$$

with $\nu=2 /(N-2)$. For later convenience we introduce

$$
\tilde{S}_{\alpha \beta}^{\delta \gamma}(\theta)=S_{\alpha \beta}^{\delta \gamma}(\theta) / S_{+}(\theta)=\tilde{b}(\theta) \delta_{\alpha}^{\gamma} \delta_{\beta}^{\delta}+\tilde{c}(\theta) \delta_{\alpha}^{\delta} \delta_{\beta}^{\gamma}+\tilde{d}(\theta) \mathbf{C}^{\delta \gamma} \mathbf{C}_{\alpha \beta}
$$

with

$$
\tilde{b}(\theta)=\frac{\theta}{\theta-i \pi \nu}, \quad \tilde{c}(\theta)=-\frac{i \pi \nu}{\theta-i \pi \nu}, \quad \tilde{d}(\theta)=-\frac{\theta}{\theta-i \pi \nu} \frac{i \pi \nu}{i \pi-\theta} .
$$


We will also need $\stackrel{\circ}{S}(z)$ the S-matrix for $O(N-2)$

$$
\tilde{\tilde{S}}_{\alpha \beta}^{\delta \gamma}(\theta)=\stackrel{\circ}{S}_{\alpha \beta}^{\delta \gamma}(\theta) / \stackrel{\circ}{S}_{+}(\theta)=\tilde{\tilde{b}}(\theta) \delta_{\alpha}^{\gamma} \delta_{\beta}^{\delta}+\tilde{\tilde{c}}(\theta) \delta_{\alpha}^{\delta} \delta_{\beta}^{\gamma}+\tilde{\tilde{d}}(\theta) \mathbf{C}^{\delta \gamma} \mathbf{C}_{\alpha \beta}
$$

where $\nu$ is replaced by $\stackrel{\nu}{=} 2 /(N-4)$.

\subsubsection{Bound states}

Following $[7,42,43]$ we write for the fundamental fermions $\alpha, \beta, \beta^{\prime}, \alpha^{\prime}$

$$
i \operatorname{Res}_{\theta=i \eta}(\sigma S)_{\alpha \beta}^{\beta^{\prime} \alpha^{\prime}}(\theta)=\sum_{\gamma} \Gamma_{\gamma}^{\beta^{\prime} \alpha^{\prime}} \Gamma_{\alpha \beta}^{\gamma}: \quad i \operatorname{Res} \gamma_{\alpha}^{\beta^{\prime}}=
$$

where $\sigma=-1$ is the statistics factor. The intertwiner $\Gamma_{\alpha \beta}^{\gamma}$ and the dual one $\Gamma_{\gamma}^{\beta \alpha}$ satisfy the crossing relation

$$
\Gamma_{\gamma}^{\beta \alpha}=\mathbf{C}_{\gamma \gamma^{\prime}} \Gamma_{\alpha^{\prime} \beta^{\prime}}^{\gamma^{\prime}} \mathbf{C}^{\beta^{\prime} \beta} \mathbf{C}^{\alpha^{\prime} \alpha}: \bigcup_{\gamma}^{\beta}=\overbrace{\gamma}^{\alpha}{ }^{\alpha}
$$

with the charge conjugation matrix $\mathbf{C}$ (2.4). Here we have for $\eta=\pi \nu$

$$
\begin{aligned}
i \operatorname{Res}_{\theta=i \pi \nu}(\sigma S)_{\alpha \beta}^{\beta^{\prime} \alpha^{\prime}}(\theta) & =-i \operatorname{Res}_{\theta=i \pi \nu} \frac{\sinh \theta+i \sin \pi \nu}{\sinh \theta-i \sin \pi \nu}\left(S^{\min }\right)_{\alpha \beta}^{\beta^{\prime} \alpha^{\prime}}(\theta) \\
& =2 \tan \pi \nu\left(S^{\min }\right)_{\alpha \beta}^{\beta^{\prime} \alpha^{\prime}}(i \pi \nu) .
\end{aligned}
$$

\section{Generalized form factors}

Form factor equations. The form factor equations for the $O(N)$ Gross-Neveu-model are similar to the ones of the $O(N) \sigma$-model in [3]. However, here there are additional statistics factors. The $F_{\alpha}^{\mathcal{O}}(\underline{\theta})$ defined by $(1.2)$ are considered as the components of a covector valued function $F_{1 \ldots n}^{\mathcal{O}}(\underline{\theta})$ which satisfies:

(i) Watson's equation

$$
F_{\ldots i j \ldots}^{\mathcal{O}}\left(\ldots, \theta_{i}, \theta_{j}, \ldots\right)=F_{\ldots j i \ldots}^{\mathcal{O}}\left(\ldots, \theta_{j}, \theta_{i}, \ldots\right)(\sigma S)_{i j}\left(\theta_{i j}\right)
$$

with $\theta_{i j}=\theta_{i}-\theta_{j}$ and $\sigma_{i j}=-1$ for fermions.

(ii) Crossing equation

$$
\begin{aligned}
&{ }^{\text {out }, \overline{1}}\left\langle\theta_{1}|\mathcal{O}(0)| \theta_{2}, \ldots, \theta_{n}\right\rangle_{2 \ldots n}^{\text {in,conn. }} \\
& \quad=F_{1 \ldots n}^{\mathcal{O}}\left(\theta_{1}+i \pi, \theta_{2}, \ldots, \theta_{n}\right) \sigma_{1}^{\mathcal{O}} \mathbf{C}^{\overline{1} 1}=F_{2 \ldots n 1}^{\mathcal{O}}\left(\theta_{2}, \ldots, \theta_{n}, \theta_{1}-i \pi\right) \mathbf{C}^{1 \overline{1}}
\end{aligned}
$$

with the charge conjugation matrix $\mathbf{C}^{\overline{1} 1}$ and the statistics factor $\sigma_{1}^{\mathcal{O}}$ of the operator $\mathcal{O}$ with respect to the particle 1. 
(iii) Recursion equation

$$
\underset{\theta_{12}=i \pi}{\operatorname{Res}} F_{1 \ldots n}^{\mathcal{O}}\left(\theta_{1}, \ldots, \theta_{n}\right)=2 i \mathbf{C}_{12} F_{3 \ldots n}^{\mathcal{O}}\left(\theta_{3}, \ldots, \theta_{n}\right)\left(\mathbf{1}-\sigma_{2}^{\mathcal{O}}(\sigma S)_{2 n} \ldots(\sigma S)_{23}\right),
$$

(iv) Because there are bound states in the model the function $F_{\underline{\alpha}}^{\mathcal{O}}(\underline{\theta})$ has additional poles. If for instance the particles 1 and 2 form a bound state (12), there is a pole at $\theta_{12}=i \eta,(0<\eta<\pi)$ such that

$$
\underset{\theta_{12}=i \eta}{\operatorname{Res}} F_{12 \ldots n}^{\mathcal{O}}\left(\theta_{1}, \theta_{2}, \ldots, \theta_{n}\right)=F_{(12) \ldots n}^{\mathcal{O}}\left(\theta_{(12)}, \ldots, \theta_{n}\right) \sqrt{2} \Gamma_{12}^{(12)}
$$

where the bound state intertwiner $\Gamma_{12}^{(12)}$ of (2.9) and the values of $\theta_{1}, \theta_{2}, \theta_{(12)}$ and $\eta$ are given in $[7,42,43]$.

(v) Lorentz covariance

$$
F_{1 \ldots n}^{\mathcal{O}}\left(\theta_{1}+\mu, \ldots, \theta_{n}+\mu\right)=e^{s \mu} F_{1 \ldots n}^{\mathcal{O}}\left(\theta_{1}, \ldots, \theta_{n}\right)
$$

if the local operator transforms under Lorentz transformations as $\mathcal{O} \rightarrow e^{s \mu} \mathcal{O}$ where $s$ is the "spin" of $\mathcal{O}$.

The statistics factors in (ii) and (iii) are not arbitrary, but consistency and crossing implies that both are the same and that the for anti-particle $\sigma_{1}^{\mathcal{O}} \sigma_{\overline{1}}^{\mathcal{O}}=1$ holds (see also [19]). In $[18,43]$ was shown that the form factor equations follow from general LSZ assumptions and "maximal analyticity".

Minimal form factors. The solutions of Watson's and the crossing equations (i) and (ii) for two particles

$$
\begin{aligned}
F(\theta) & =S(\theta) F(-\theta) \\
F(i \pi-\theta) & =F(i \pi+\theta)
\end{aligned}
$$

with no poles in the physical strip $0 \leq \operatorname{Im} \theta \leq \pi$ and at most a simple zero at $\theta=0$ are the minimal form factors $[6]$

$$
\begin{aligned}
& F_{+}^{\min }(\theta)=\exp \int_{0}^{\infty} \frac{d t}{t \sinh t} \frac{e^{-t(1-\nu)}-e^{-t}}{1+e^{-t}}\left(1-\cosh t\left(1-\frac{\theta}{i \pi}\right)\right) \\
& F_{-}^{\min }(\theta)=\frac{\cosh \frac{1}{2}(i \pi-\theta) \Gamma^{2}\left(\frac{1}{2}+\frac{1}{2} \nu\right)}{\Gamma\left(1+\frac{1}{2} \nu-\frac{1}{2 \pi i} \theta\right) \Gamma\left(\frac{1}{2} \nu+\frac{1}{2 \pi i} \theta\right)} F_{+}^{\min }(\theta) \\
& F_{0}^{\min }(\theta)=\frac{2 \tanh \frac{1}{2}(i \pi-\theta)}{i \pi-\theta} F_{-}^{\min }(\theta) .
\end{aligned}
$$

They belong to the S-matrix eigenvalues $S_{ \pm}=b \pm c$ and $S_{0}=b+c+N d$ (see (2.3)). For the construction of the off-shell Bethe Ansatz the minimal solution of the form factor equation (3.1) for the highest weight eigenvalue of the $O(N)$ S-matrix

$$
F(\theta)=\sigma S_{+}(\theta) F(-\theta)=-a(\theta) F(-\theta)
$$


is essential. We take the solution ${ }^{3}$

$$
\begin{aligned}
F(\theta) & =c \cosh \frac{1}{2}(i \pi-\theta) F_{+}^{\min }(\theta) \\
& =c \exp \left(\int_{0}^{\infty} \frac{d t}{t \sinh t} \frac{1+e^{-t(1-\nu)}}{1+e^{-t}}\left(1-\cosh t\left(1-\frac{\theta}{i \pi}\right)\right)\right)
\end{aligned}
$$

or $^{4}$

$$
F(\theta)=\frac{G\left(\frac{1}{2} \frac{\theta}{i \pi}\right) G\left(1-\frac{1}{2} \frac{\theta}{i \pi}\right)}{G\left(\frac{1}{2}+\frac{1}{2} \frac{\theta}{i \pi}\right) G\left(\frac{3}{2}-\frac{1}{2} \frac{\theta}{i \pi}\right)} \frac{G\left(\frac{1}{2}-\frac{1}{2} \nu+\frac{1}{2} \frac{\theta}{i \pi}\right) G\left(\frac{3}{2}-\frac{1}{2} \nu-\frac{1}{2} \frac{\theta}{i \pi}\right)}{G\left(1-\frac{1}{2} \nu+\frac{1}{2} \frac{\theta}{i \pi}\right) G\left(2-\frac{1}{2} \nu-\frac{1}{2} \frac{\theta}{i \pi}\right)}
$$

where $G(z)$ is Barnes G-function, which satisfies (see e.g. [44])

$$
G(1+z)=\Gamma(z) G(z) .
$$

For convenience we have introduced the constant $c$ (see (4.9))

$$
c=G^{2}\left(\frac{1}{2}\right) G^{2}\left(1-\frac{1}{2} \nu\right) G^{-2}\left(\frac{3}{2}-\frac{1}{2} \nu\right) .
$$

The full 2-particle form factors are

$$
F_{+,-, 0}(\theta)=\frac{-\cos ^{2} \frac{1}{2} \pi \nu}{\sinh \frac{1}{2}(\theta-i \pi \nu) \sinh \frac{1}{2}(\theta+i \pi \nu)} F_{+,-, 0}^{\min }(\theta) .
$$

They are non-minimal solutions of (3.6) containing the bound state pole at $\theta=i \pi \nu$ (see $(2.16)$ of $[6])$.

\section{$4 O(N)$ form factors and Bethe Ansatz}

\subsection{The fundamental theorem}

Following [6] we write the general form factor $F_{1 \ldots n}^{\mathcal{O}}(\underline{\theta})$ for n-fundamental particles as (1.3) where $F(\theta)$ is the minimal form factor function $(3.11)$. The $\mathrm{K}$-function $K_{1 \ldots n}^{\mathcal{O}}(\underline{\theta})$ is determined by the form factor equations (i) $-(\mathrm{v})$. We propose the K-function in terms of a nested 'off-shell' Bethe Ansatz (1.4) as a multiple contour integral.

The basic Bethe Ansatz co-vectors in (1.5) are defined as (for more details see [1, 3])

$$
\tilde{\Phi^{\alpha}} \underline{\dot{\beta}}(\underline{\theta}, \underline{z})=\left(\Pi_{\underline{\beta}}^{\underline{\beta}}(\underline{z}) \Omega \tilde{T}_{1}^{\beta_{m}}\left(\underline{\theta}, z_{m}\right) \ldots \tilde{T}_{1}^{\beta_{1}}\left(\underline{\theta}, z_{1}\right)\right)_{\underline{\alpha}} .
$$

The matrix $\Pi \underline{\underline{\beta}}(\underline{\underline{z}})$ intertwines ${ }^{5}$ between the S-matrices $S$ of $O(N)$ and $\stackrel{\circ}{S}$ of $O(N-2)$

$$
\tilde{\tilde{S}}_{i j}\left(z_{i j} \stackrel{\circ}{\nu} / \nu\right) \Pi_{\ldots i j \ldots}(\underline{z})=\Pi_{\ldots j i \ldots}(\underline{z}) \tilde{S}_{i j}\left(z_{i j}\right) .
$$

\footnotetext{
${ }^{3}$ The minus sign in (3.10) and the factor $\cosh \frac{1}{2}(i \pi-\theta)$ is due to fermionic statistics of the fundamental particles (see also eq. 4.14 of [18]).

${ }^{4}$ Private communication: Karol K. Kozlowski pointed out to one of the authors (M.K.), that the minimal form factors may be expressed in terms of Barnes G-function.

${ }^{5}$ This matrix $\Pi$ is trivial for the $\mathrm{SU}(N)$ Bethe Ansatz because the $\mathrm{SU}(N)$ S-matrix does not depend on $N$ for a suitable normalization and parametrization.
} 
The Bethe Ansatz co-vectors (4.1) are generalizations of vectors introduced by Tarasov [45] for the Korepin-Izergin model. Below we will use the following relations for special components of $\Pi$ (for more details see $[1-3]$ )

$$
\underline{\Pi^{\beta}} \underline{\underline{\beta}}= \begin{cases}0 & \text { for } \beta_{1}=1, \text { or } \beta_{m}=\overline{1} \\ \delta_{\beta_{1}}^{\dot{\beta}_{1}} \Pi_{\beta_{2} \ldots \beta_{m}}^{\dot{\beta}_{2} \ldots \dot{\beta}_{m}} & \text { for } \beta_{1} \neq \overline{1} \\ \Pi_{\dot{\beta}_{1} \ldots \dot{\beta}_{m-1}}^{\beta_{1} \ldots \beta_{m-1}} \delta_{\beta_{m}}^{\dot{\beta}_{m}} & \text { for } \beta_{m} \neq 1 .\end{cases}
$$

The scalar function $\tilde{h}(\underline{\theta}, \underline{z})$ in (1.4) depends only on the S-matrix and not on the specific operator $\mathcal{O}(x)$

$$
\tilde{h}(\underline{\theta}, \underline{z})=\prod_{i=1}^{n} \prod_{j=1}^{m} \tilde{\phi}\left(\theta_{i}-z_{j}\right) \prod_{1 \leq i<j \leq m} \tau\left(z_{i}-z_{j}\right) .
$$

The functions $\tilde{\phi}$ and $\tau$ satisfy the shift equations

$$
\begin{aligned}
\tilde{\phi}(\theta-2 \pi i) & =-\tilde{b}(\theta) \tilde{\phi}(\theta) \\
\tau(z-2 \pi i) / \tilde{b}(2 \pi i-z) & =\tau(z) / \tilde{b}(z)
\end{aligned}
$$

which are related to the form factor equation (ii) or (3.2) [1-3]. Here for the $O(N)$ GrossNeveu model

where $\tilde{\phi}(\theta)$ is ${ }^{6}$

$$
\tau(z)=\frac{1}{\tilde{\phi}(-z) \tilde{\phi}(z)}
$$

$$
\tilde{\phi}(\theta)=\Gamma\left(1-\frac{1}{2} \nu+\frac{1}{2 \pi i} \theta\right) \Gamma\left(-\frac{1}{2 \pi i} \theta\right) .
$$

The form factor equation (iii) or (3.3) (as will be discussed in appendix A) requires that

$$
F(\theta) F(\theta+i \pi) \tilde{\phi}(-\theta-i \pi+i \pi \nu) \tilde{\phi}(-\theta)=1 .
$$

The function (4.8) satisfies this relation. Notice that the equations (4.8) and (4.9) also determine the normalization constant $c$ in (3.11) and (3.12).

Similar as in [3] the integration contours $\mathcal{C}_{\underline{\theta}}^{(j)}$ in (1.4) depend on whether $j$ is even or odd, they are depicted in figure 3. The Ansatz (1.3) and (1.4) transforms the matrix equations (i)-(v) (see (3.2)-(3.5)) into much simpler scalar equations for the scalar pfunction $p^{\mathcal{O}}(\underline{\theta}, \underline{z})$. This function depends on the specific operator $\mathcal{O}(x)$ and is in general a simple function of $e^{\theta_{i}}$ and $e^{z_{j}}$.

Theorem 1 Assume that:

1. The p-function $p^{\mathcal{O}}(\underline{\theta}, \underline{z})$ satisfies the equations

$$
\left.\begin{array}{l}
\left(\mathrm{i}^{\prime}\right) p^{\mathcal{O}}(\underline{\theta}, \underline{z}) \text { is symmetric under } \theta_{i} \leftrightarrow \theta_{j} \\
\left(\mathrm{ii}_{1}^{\prime}\right) p^{\mathcal{O}}(\underline{\theta}, \underline{z})=\sigma^{\mathcal{O}}(-1)^{m} p^{\mathcal{O}}\left(\theta_{1}+2 \pi i, \theta_{2}, \ldots, \underline{z}\right) \\
\left(\mathrm{ii}_{2}^{\prime}\right) p^{\mathcal{O}}(\underline{\theta}, \underline{z})=(-1)^{n} p^{\mathcal{O}}\left(\underline{\theta}, z_{1}+2 \pi i, z_{2}, \ldots\right) \\
\left(\mathrm{iii}^{\prime}\right) p^{\mathcal{O}}(\underline{\theta}, \underline{z})=p^{\mathcal{O}}(\underline{\underline{\theta}}, \underline{\check{z}})
\end{array}\right\}
$$

where in (iii') $\theta_{12}=i \pi, \quad z_{1}=\theta_{1}-i \pi \nu$ and $z_{2}=\theta_{2}$. The short notations $\underline{\ddot{\theta}}=$ $\left(\theta_{3}, \ldots, \theta_{n}\right)$ and $\underline{\check{z}}=\left(z_{3}, \ldots, z_{m}\right)$ are used.

\footnotetext{
${ }^{6}$ This is in contrast to the $\sigma$-model case where the $\tilde{\phi}$-functions depend on whether $j$ in (4.4) is even or odd.
} 


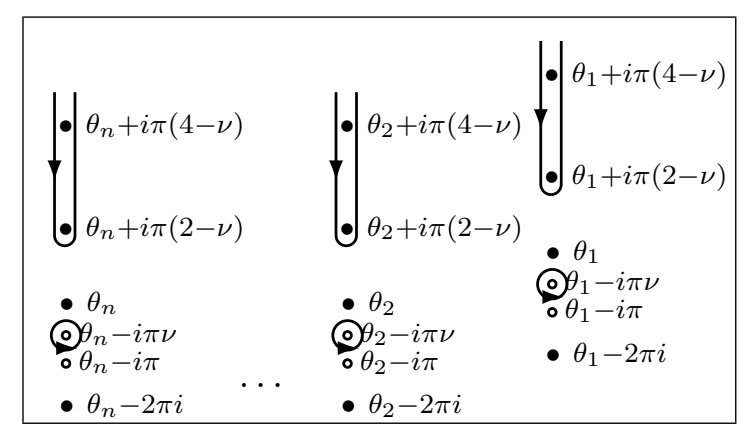

(odd)

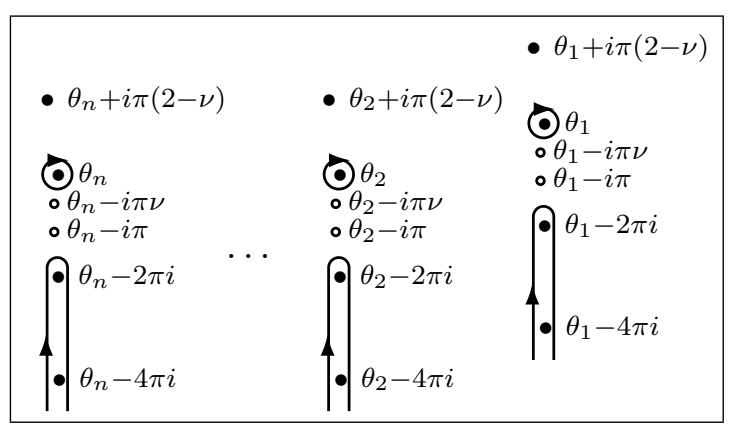

(even)

Figure 3. The integration contours $\mathcal{C}_{\theta}^{(o)}$ and $\mathcal{C}_{\theta}^{(e)}$. The bullets refer to poles of the integrand resulting from $\tilde{\phi}\left(\theta_{i}-z_{j}\right)$ and the small open circles refer to poles originating from $\tilde{S}\left(\theta_{i}-z_{j}\right)$.

2. The higher level function $L_{\beta}(\underline{z})$ in (1.5) satisfies $(\mathrm{i})^{(k)}-(\mathrm{iii})^{(k)}$ of (4.13)-(4.15) for $k=1$.

3. A suitable choice of the normalization constants in (1.4).

Then the co-vector valued function $F_{\underline{\alpha}}(\underline{\theta})$ given by the Ansatz (1.3) and the integral representation (1.4) satisfies the form factor equations (i)-(v) of (3.1)-(3.5).

The proof of this theorem can be found in appendix A.

\subsection{Higher level off-shell Bethe Ansatz}

For this discussion it is convenient to introduce the variables $u, v$ defined by $\theta=i \pi \nu_{k} u, z=$ $i \pi \nu_{k} v$ and $\nu_{k}=2 /(N-2 k-2)$. For the $O(N-2 k)$ S-matrix $S^{(k)}(u)$ we write as in (2.6)

$$
\begin{aligned}
\tilde{S}^{(k)}(u) & =S^{(k)} / S_{+}^{(k)}=\tilde{b}(u) \mathbf{1}+\tilde{c}(u) \mathbf{P}+\tilde{d}_{k}(u) \mathbf{K} \\
\tilde{b}(u) & =\frac{u}{u-1}, \quad \tilde{c}(u)=\frac{-1}{u-1}, \quad \tilde{d}_{k}(u)=\frac{u}{u-1} \frac{1}{u-1 / \nu_{k}} .
\end{aligned}
$$

and define

$$
\begin{gathered}
K_{\underline{\alpha}}^{(k)}(\underline{u})=\tilde{N}_{m_{k}}^{(k)} \int_{\mathcal{C}_{\underline{u}}^{(1)}} d v_{1} \cdots \int_{\mathcal{C}_{\underline{u}}^{\left(m_{k}\right)}} d v_{m_{k}} \tilde{h}(\underline{u}, \underline{v}) p^{(k)}(\underline{u}, \underline{v}) \tilde{\Psi}_{\underline{\alpha}}^{(k)}(\underline{u}, \underline{v}) \\
\tilde{\Psi}_{\underline{\alpha}}^{(k)}(\underline{u}, \underline{v})=L_{\underline{\hat{\beta}}}^{(k)}(\underline{v})\left(\tilde{\Phi}^{(k)}\right)_{\underline{\alpha}}^{\underline{\underline{\beta}}}(\underline{u}, \underline{v}), \quad L_{\underline{\hat{\beta}}}^{(k)}(\underline{v})=K_{\underline{\hat{\beta}}}^{(k+1)}(\underline{v})
\end{gathered}
$$

with $\underline{u}=u_{1}, \ldots, u_{n_{k}}, \underline{v}=v_{1}, \ldots, v_{m_{k}}$ and $m_{k}=n_{k+1}$. 
The equations $(\mathrm{i})^{(k)}-(\mathrm{iii})^{(k)}$ for $k>0$ are in terms of these variables similar as in [3]

$(\mathrm{i})^{(k)}$

$$
K_{\ldots i j \ldots}^{(k)}\left(\ldots, u_{i}, u_{j}, \ldots\right)=K_{\ldots j i \ldots}^{(k)}\left(\ldots, u_{j}, u_{i}, \ldots\right) \tilde{S}_{i j}^{(k)}\left(u_{i j}\right)
$$

$\left(\right.$ ii) ${ }^{(k)}$

$$
K_{1 \ldots n_{k}}^{(k)}\left(u_{1}+2 / \nu, u_{2}, \ldots, u_{n_{k}}\right) \mathbf{C}^{\overline{1} 1}=K_{2 \ldots n_{k} 1}^{(k)}\left(u_{2}, \ldots, u_{n_{k}}, u_{1}\right) \mathbf{C}^{1 \overline{1}}
$$

$(\text { iii) })^{(k)}$

$$
\underset{u_{12}=1 / \nu_{k}}{\operatorname{Res}} K_{1 \ldots n_{k}}^{(k)}\left(u_{1}, \ldots, u_{n_{k}}\right)=\prod_{i=3}^{n_{k}} \tilde{\phi}\left(u_{i 1}+1\right) \tilde{\phi}\left(u_{i 2}\right) \mathbf{C}_{12} K_{3 \ldots n_{k}}^{(k)}\left(u_{3}, \ldots, u_{n_{k}}\right)
$$

in addition we have here the bound state relation

$(\text { iv })^{(k)}$

$$
\underset{u_{12}=1}{\operatorname{Res}} F_{12 \ldots n}^{(k)}\left(u_{1}, u_{2}, \ldots, u_{n}\right)=F_{(12) \ldots n}^{(k)}\left(u_{(12)}, \ldots, u_{n}\right) \sqrt{2} \Gamma_{12}^{(12)} .
$$

The form factor equations (i)-(iv) of (3.1)-(3.4) for $O(N-2 k)$ are similar to these higher level equations. There are, however, two differences: 1) The shift in (ii) ${ }^{(k)}$ is the one of $O(N)$ but not that of $O(N-2 k)$. 2) There is only one term on the right hand side in $(\text { iii) })^{(k)}$.

We assume that the p-function $p^{(k)}(\underline{u}, \underline{v})$ satisfies the equations

(i') $p^{(k)}(\underline{u}, \underline{v})$ is symmetric under $u_{i} \leftrightarrow u_{j}, v_{i} \leftrightarrow v_{j}$

(ii') $p^{(k)}(\underline{u}, \underline{v})=(-1)^{m_{k}} p^{(k)}\left(u_{1}+2 / \nu, u_{2}, \ldots, \underline{v}\right)=(-1)^{n_{k}} p^{(k)}\left(\underline{u}, v_{1}+2 / \nu, v_{2}, \ldots\right)$

(iii') $p^{(k)}(\underline{u}, \underline{z})=p^{(k)}(\underline{\underline{u}}, \underline{\underline{y}})$ for $u_{12}=1 / \nu_{k}, v_{1}=u_{1}-1$ and $v_{2}=u_{2}$

where we use the short notations $\underline{\check{u}}=\left(u_{3}, \ldots, u_{n_{k}}\right)$ and $\underline{\check{v}}=\left(v_{3}, \ldots, v_{m_{k}}\right)$.

Lemma 2 For $0<k<\frac{1}{2}(N-4)$ the functions $K_{\underline{\alpha}}^{(k)}(\underline{u})$ of (4.12) satisfy the equations $(\mathrm{i})^{(k)}$, (ii) ${ }^{(k)}$ and (iii) $)^{(k)}$, if the corresponding relations are satisfied for $K_{\underline{\underline{\beta}}}^{(k+1)}(\underline{v})$ and if suitable choice of the normalization constants in (4.12) is assumed. The weights of the operator $\mathcal{O}$

$$
w^{\mathcal{O}}=\left(w_{1}, \ldots, w_{N / 2}\right)=\left(n_{0}-n_{1}, \ldots, n_{N / 2-2}-n_{-}-n_{+}, n_{-}-n_{+}\right)
$$

determine the numbers $m_{k}=n_{k+1}$ for a given number of particles $n=n_{0}$

The proof of this lemma can be found in appendix C.1. 


\section{$5 \quad$ Examples}

In this section, to illustrate our general results we present some simple examples.

\subsection{Current}

The $O(N)$ Noether current ${ }^{7}$

$$
J_{\mu}^{\alpha \beta}=\bar{\psi}^{\alpha} \gamma_{\mu} \psi^{\beta}
$$

transforms as the antisymmetric tensor representation of $O(N)$. This operator has therefore the weights $w^{J}=\left(w_{1}, \ldots, w_{N / 2}\right)=(1,1,0, \ldots, 0)$ (see $\left.[1,2]\right)$, which implies with (4.18) that

$$
n-2=n_{1}-1=n_{2}=\cdots=n_{N / 2-2}=n_{-}+n_{+}, n_{-}=n_{+} .
$$

where $n_{i}$ are the numbers of integrations in the various levels of the off-shell Bethe Ansatz. The existence of a pseudo-potential $J^{\alpha \beta}(x)$ follows from the conservation law $\partial^{\mu} J_{\mu}^{\alpha \beta}=0$

$$
J_{\mu}^{\alpha \beta}(x)=\epsilon_{\mu \nu} \partial^{\nu} J^{\alpha \beta}(x) .
$$

For the form factors of both operators we have the relation

$$
F_{\underline{\alpha}}^{J_{\mu}}(\underline{\theta})=-i \epsilon_{\mu \nu}\left(\sum p_{i}^{\nu}\right) F_{\underline{\alpha}}^{J}(\underline{\theta}) .
$$

Because the Bethe Ansatz yields highest weight states we obtain the matrix elements of the highest weight component of $J^{\alpha \beta}$ which means in the complex basis $J(x)=J^{12}(x)$.

We propose the form factors of the operator $J(x)$ (for $n=m+1=n_{1}+1=n_{2}+2$ even)

$$
\begin{aligned}
\langle 0|J(0)| \underline{\theta}\rangle_{\underline{\alpha}} & =F_{\underline{\alpha}}^{J}(\underline{\theta})=\prod_{i<j} F\left(\theta_{i j}\right) K_{\underline{\alpha}}^{J}(\underline{\theta}) \\
K_{\underline{\alpha}}^{J}(\underline{\theta}) & =N_{n}^{J} \int_{\mathcal{C}_{\underline{\theta}}^{(1)}} d z_{1} \ldots \int_{\mathcal{C}_{\underline{\theta}}^{(m)}} d z_{m} \tilde{h}(\underline{\theta}, \underline{z}) p^{J}(\underline{\theta}, \underline{z}) \tilde{\Psi}_{\underline{\alpha}}(\underline{\theta}, \underline{z}) .
\end{aligned}
$$

Expressing $\tilde{\Psi}_{\underline{\alpha}}(\underline{\theta}, \underline{z})$ in terms of all higher level Bethe Ansatzes there appears the product of all level p-functions $p^{J}(\underline{\theta}, \underline{z})$. For the example of the current it depends on the $\theta_{i}$ and the second level $z_{j}^{(2)}$

$$
p^{J}(\underline{\theta}, \underline{z})=e^{\frac{1}{2}\left(\sum_{i=1}^{n} \theta_{i}-\sum_{j=1}^{n_{2}} z_{j}^{(2)}-\frac{1}{2} n_{2} i \pi \stackrel{\nu}{ }\right)} / \sum_{i=1}^{n} e^{\theta_{i}}+e^{-\frac{1}{2}\left(\sum_{i=1}^{n} \theta_{i}-\sum_{j=1}^{n_{2}} z_{j}^{(2)}-\frac{1}{2} n_{2} i \pi \nu\right)} / \sum_{i=1}^{n} e^{-\theta_{i}}
$$

which satisfies (4.10) with

$$
\begin{array}{ll}
\text { charge } & Q^{J}=0 \\
\text { weight vector } & w^{J}=(1,1,0, \ldots, 0) \\
\text { statistics factor } & \sigma^{J}=1 \\
\text { spin } & s^{J}=0, s^{J_{\mu}}=1 .
\end{array}
$$

\footnotetext{
${ }^{7}$ In the real basis.
} 
For example for 2-particle form factor we obtain (see appendix B)

$$
\begin{aligned}
& F_{\alpha_{1} \alpha_{2}}^{J^{\alpha \beta}}\left(\theta_{1}, \theta_{2}\right)=i m\left(\delta_{\alpha_{1}}^{\alpha} \delta_{\alpha_{2}}^{\beta}-\delta_{\alpha_{1}}^{\beta} \delta_{\alpha_{2}}^{\alpha}\right) \frac{1}{\cosh \frac{1}{2} \theta_{12}} F_{-}(\theta) \\
& F_{\alpha_{1} \alpha_{2}}^{J_{\mu}^{\alpha \beta}}\left(\theta_{1}, \theta_{2}\right)=i\left(\delta_{\alpha_{1}}^{\alpha} \delta_{\alpha_{2}}^{\beta}-\delta_{\alpha_{1}}^{\beta} \delta_{\alpha_{2}}^{\alpha}\right) \bar{v}\left(\theta_{1}\right) \gamma_{\mu} u\left(\theta_{2}\right) F_{-}(\theta)
\end{aligned}
$$

with $F_{-}(\theta)$ of $(3.13)$ and $(3.8), \bar{v}\left(\theta_{1}\right) \gamma^{ \pm} u\left(\theta_{2}\right)= \pm i 2 m e^{ \pm \frac{1}{2}\left(\theta_{1}+\theta_{2}\right)}$ and (5.1). This result agrees with $[6]$.

\section{$5.2 \quad$ Field}

For the fundamental field $\psi^{\alpha}(x)$ in (1.1) the numbers $n_{i}$ of integrations in the various levels of the off-shell Bethe Ansatz satisfy

$$
n-1=n_{1}=n_{2}=\cdots=n_{N / 2-2}=n_{-}+n_{+}, n_{-}=n_{+}
$$

because $\psi^{\alpha}$ transforms as the vector representation of $O(N)$ (see [1,2] and (4.18)). We restrict to component $\psi=\psi^{1}$ because as usual the Bethe Ansatz yields highest weight states. For convenience we multiply the field with the Dirac operator and take

$$
\chi(x)=i(-i \gamma \partial+m) \psi(x)
$$

We propose for the $n$-particle form factors $\left(n=m+1\right.$ odd) for the spinor components $\chi^{( \pm)}$

$$
\begin{aligned}
\left\langle 0\left|\chi^{( \pm)}(0)\right| \underline{\theta}\right\rangle_{\underline{\alpha}} & =F_{\underline{\alpha}}^{\chi^{( \pm)}}(\underline{\theta})=\prod_{i<j} F\left(\theta_{i j}\right) K_{\underline{\alpha}}^{\chi^{( \pm)}}(\underline{\theta}) \\
K_{\underline{\alpha}}^{\chi^{( \pm)}}(\underline{\theta}) & =N_{n}^{\chi} \int_{\mathcal{C}_{\underline{\theta}}^{(1)}} d z_{1} \ldots \int_{\mathcal{C}_{\underline{\theta}}^{(m)}} d z_{m} \tilde{h}(\underline{\theta}, \underline{z}) p^{\chi^{( \pm)}}(\underline{\theta}, \underline{z}) \tilde{\Psi}_{\underline{\alpha}}(\underline{\theta}, \underline{z})
\end{aligned}
$$

with the p-function (for $n=m+1=$ odd $>1$ )

$$
p^{\chi^{( \pm)}}(\underline{\theta}, \underline{z})=\exp \left(\mp \frac{1}{2}\left(\sum_{j=1}^{n} \theta_{j}-\sum_{j=1}^{m} z_{j}-\frac{1}{2} m i \pi \nu\right)\right)
$$

which solves (4.10) with

$$
\begin{array}{lll}
\text { charge } & Q^{\psi}=1 \\
\text { weight vector } & w^{\psi}=(1, \ldots, 0) \\
\text { statistics factor } & \sigma^{\psi}=-1 \\
\text { spin } & s^{\psi}=\frac{1}{2}
\end{array}
$$

The one particle form factor is trivial

$$
\langle 0|\psi(0)| \theta\rangle_{\alpha}=F_{\alpha}^{\psi}(\theta)=\delta_{\alpha}^{1} u(\theta) .
$$


For the three particle form factor $(n=3, m=2)$ the equations (5.6) and (5.7) write as

$$
\begin{aligned}
\langle 0|\chi(0)| \underline{\theta}\rangle_{\underline{\alpha}} & =F_{\underline{\alpha}}^{\chi}(\underline{\theta})=F\left(\theta_{12}\right) F\left(\theta_{13}\right) F\left(\theta_{23}\right) K_{\underline{\alpha}}^{\chi}(\underline{\theta}) \\
K_{\underline{\alpha}}^{\chi}(\underline{\theta}) & =N_{3}^{\chi} \int_{\mathcal{C}_{\underline{\theta}}^{(o)}} d z_{1} \int_{\mathcal{C}_{\underline{\underline{\theta}}}^{(e)}} d z_{2} \tilde{h}(\underline{\theta}, \underline{z}) p^{\chi}(\underline{\theta}, \underline{z}) \tilde{\Psi}_{\underline{\alpha}}(\underline{\theta}, \underline{z})
\end{aligned}
$$

with

$$
\begin{aligned}
\tilde{h}(\underline{\theta}, \underline{z}) & =\prod_{i=1}^{3} \tilde{\phi}\left(\theta_{i}-z_{1}\right) \tilde{\phi}\left(\theta_{i}-z_{2}\right) \frac{1}{\tilde{\phi}\left(z_{12}\right) \tilde{\phi}\left(-z_{12}\right)} \\
p^{\chi^{ \pm}}(\underline{\theta}, \underline{z}) & =e^{\mp \frac{1}{2}\left(\theta_{1}+\theta_{2}+\theta_{3}-z_{1}-z_{2}-i \pi \nu\right)} \\
\tilde{\Psi}_{\underline{\alpha}}(\underline{\theta}, \underline{z}) & =L_{\underline{\beta}}(\underline{z})\left(\Pi_{\underline{\beta}}^{\underline{\beta}}(\underline{z}) \Omega \tilde{T}_{1}^{\beta_{2}}\left(\underline{\theta}, z_{2}\right) \tilde{T}_{1}^{\beta_{1}}\left(\underline{\theta}, z_{1}\right)\right)_{\underline{\alpha}} .
\end{aligned}
$$

Lemma 2 for the $O(N-2)$ weights $w=(0, \ldots, 0)$ yields for the higher level function $L_{\dot{\beta}_{1} \dot{\beta}_{2}}(\underline{z})={\stackrel{\mathbf{C}}{\dot{\beta}_{1} \dot{\beta}_{2}}}_{L} L\left(z_{12}\right)$ with

$$
L(z)=\frac{\Gamma\left(1-\frac{1}{2} \nu-\frac{z}{2 \pi i}\right) \Gamma\left(-\frac{1}{2} \nu+\frac{z}{2 \pi i}\right)}{\Gamma\left(1+\frac{1}{2}(1-\nu)-\frac{z}{2 \pi i}\right) \Gamma\left(\frac{1}{2}(1-\nu)+\frac{z}{2 \pi i}\right)}
$$

(see appendix C.2). We could not perform the integrations ${ }^{8}$ in (5.9) for general $N$, but we expand the exact expression in $1 / N$-expansion to compare the result with the $1 / N$ expansion of the $O(N)$ Gross-Neveu model in terms of Feynman graphs.

1/N expansion. We obtain the 3-particle form factor of $\chi(x)$ up to $O\left(N^{-2}\right)$ as (see appendix E.1)

$$
F_{\alpha \beta \gamma}^{\chi^{\delta}}=\frac{8 \pi m}{N}\left(\delta_{\gamma}^{\delta} \mathbf{C}_{\alpha \beta} \frac{\cosh \frac{1}{2} \theta_{12}}{\theta_{12}-i \pi} u\left(\theta_{3}\right)-\delta_{\beta}^{\delta} \mathbf{C}_{\alpha \gamma} \frac{\cosh \frac{1}{2} \theta_{13}}{\theta_{13}-i \pi} u\left(\theta_{2}\right)+\delta_{\alpha}^{\delta} \mathbf{C}_{\beta \gamma} \frac{\cosh \frac{1}{2} \theta_{23}}{\theta_{23}-i \pi} u\left(\theta_{1}\right)\right)
$$

which agrees with the $1 / N$ expansion using Feynman graphs (see appendix E.2).

Bound state form factor of $\psi$. We discuss the bound state fusion of 2 fundamental fermions $f+f \rightarrow b_{2}$, a boson of mass $m_{2}$ (see (2.2)). Writing (5.5) as

$$
\psi(x)=(i \gamma \partial+m) \tilde{\chi}(x), \quad \tilde{\chi}(x)=-i\left(\square+m^{2}\right)^{-1} \chi(x),
$$

we apply the form factor equation (iv), i.e. (3.4)

$$
\underset{\theta_{12}=i \pi \nu}{\operatorname{Res}} F_{123}^{\mathcal{O}}\left(\theta_{1}, \theta_{2}, \theta_{3}\right)=F_{(12) 3}^{\mathcal{O}}\left(\theta_{(12)}, \theta_{3}\right) \sqrt{2} \Gamma_{12}^{(12)}
$$

to the operator $\mathcal{O}=\tilde{\chi}^{9}$

$$
\underset{\theta_{12}=i \pi \nu}{\operatorname{Res}} F_{1 \overline{1} 1}^{\tilde{\chi}^{( \pm)}}(\underline{\theta})=F_{b_{2} 1}^{\tilde{\chi}^{( \pm)}}\left(\theta_{0}, \theta_{3}\right) \sqrt{2} \Gamma_{1 \overline{1}}^{b_{2}}
$$

\footnotetext{
${ }^{8}$ Doing one integral we obtain a generalization of Meijer's G-functions. The second integration does not yield known functions (to our knowledge). One could, of course, apply numerical integration techniques and determine the asymptotic behavior for large $\theta^{\prime}$ s which is under investigation [46].

${ }^{9}$ Strictly speaking $F_{1 \overline{1} 1}^{\tilde{\chi}} \pm F_{\overline{1} 11}^{\tilde{\chi}}$ give $F_{b_{2}^{(0,2)} 1}^{\tilde{\chi}}$.
} 


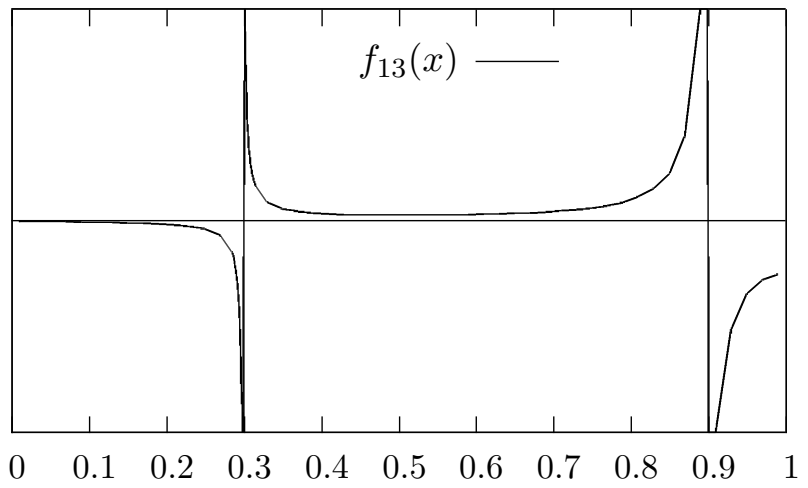

Figure 4. Plot of the bound state form factor function $f_{13}(x),(\theta=i \pi x)$ for $N=12(\nu=1 / 5)$.

The result may be written as

$$
\begin{aligned}
F_{b_{2} 1}^{\tilde{\chi}^{( \pm)}}\left(\theta_{0}, \theta_{3}\right)= & e^{\mp \frac{1}{2} \theta_{0}}\left(e^{ \pm \frac{1}{4} i \pi \nu} f_{13}\left(\theta_{03}\right)+e^{\mp \frac{1}{4} i \pi \nu} f_{32}\left(\theta_{03}\right)\right) \\
& +e^{\mp \frac{1}{2} \theta_{3}}\left(e^{ \pm \frac{1}{2} i \pi \nu} f_{11}\left(\theta_{03}\right)+e^{\mp \frac{1}{2} i \pi \nu} f_{22}\left(\theta_{03}\right)\right) .
\end{aligned}
$$

where the functions $f_{i j}$ may be calculated in terms of hypergeometric functions ${ }_{3} F_{2}$ (for more details see appendix D). For example $f_{13}$ is plotted for $N=12$ in figure 4 .

The pole at $\theta=\frac{3}{2} i \pi \nu$ (here $x=0.3$ ) belongs to the bound state fusion $b_{2}^{(r)}+f \rightarrow b_{3}^{(r \pm 1)}$, a fermion of mass $m_{3}$ (see (2.2)). The pole at $\theta=i \pi\left(1-\frac{1}{2} \nu\right)$ (here $x=0.9$ ) belongs to the bound state fusion $b_{2}^{(r)}+f \rightarrow f$, which is again the fundamental fermion. These are examples of the general "bootstrap principal" [43].

\subsection{Energy momentum}

The energy momentum tensor is in terms of fields is

$$
T^{\mu \nu}(x)=\frac{1}{2} i \bar{\psi} \gamma^{\mu} \overleftrightarrow{\partial^{\nu}} \psi-g^{\mu \nu} \mathcal{L}
$$

with the trace

$$
T_{\mu}^{\mu}(x)=m \bar{\psi} \psi .
$$

Because $T^{\mu \nu}$ is an $O(N)$ iso-scalar we have the weights $w=\left(w_{1}, \ldots, w_{N / 2}\right)=(0, \ldots, 0)$ (see $[1,2])$ which implies that

$$
n=n_{1}=\cdots=n_{N / 2-2}=n_{-}+n_{+}, n_{-}=n_{+} .
$$

We write the energy momentum tensor in terms of an energy momentum potential (see e.g. [3])

$$
\begin{aligned}
T^{\mu \nu}(x) & =R^{\mu \nu}\left(i \partial_{x}\right) T(x) \\
R^{\mu \nu}(P) & =-P^{\mu} P^{\nu}+g^{\mu \nu} P^{2} \\
T_{\mu}^{\mu}(x) & =\left(i \partial_{x}\right)^{2} T(x) .
\end{aligned}
$$


For $\bar{\psi} \psi$ we propose the $n$-particle form factor as

$$
\begin{aligned}
\langle 0|\bar{\psi} \psi(0)| \underline{\theta}\rangle_{\underline{\alpha}} & =F_{\underline{\alpha}}^{\bar{\psi} \psi}(\underline{\theta})=N_{n}^{\bar{\psi} \psi} \prod_{i<j} F\left(\theta_{i j}\right) K_{\underline{\alpha}}^{\bar{\psi} \psi}(\underline{\theta}) \\
K_{\underline{\alpha}}^{\bar{\psi} \psi}(\underline{\theta}) & =\int_{\mathcal{C}_{\underline{\theta}}^{(1)}} d z_{1} \ldots \int_{\mathcal{C}_{\underline{\theta}}^{(m)}} d z_{m} \tilde{h}(\underline{\theta}, \underline{z}) p^{\bar{\psi} \psi}(\underline{\theta}, \underline{z}) \tilde{\Psi}_{\underline{\alpha}}(\underline{\theta}, \underline{z})
\end{aligned}
$$

with $m=n=$ even and

$$
\begin{aligned}
\tilde{h}(\underline{\theta}, \underline{z}) & =\prod_{i=1}^{n} \prod_{j=1}^{m} \tilde{\phi}\left(\theta_{i}-z_{j}\right) \prod_{1 \leq i<j \leq m} \tau\left(z_{i j}\right), \\
p^{\bar{\psi} \psi}(\underline{\theta}, \underline{z}) & =1 \\
\tilde{\Psi}_{\underline{\alpha}}(\underline{\theta}, \underline{z}) & =L_{\underline{\hat{\beta}}}(\underline{z})\left(\Pi \underline{\underline{\beta}}(\underline{z}) \Omega \tilde{T}_{1}^{\beta_{m}}\left(\underline{\theta}, z_{m}\right) \ldots \tilde{T}_{1}^{\beta_{1}}\left(\underline{\theta}, z_{1}\right)\right)_{\underline{\alpha}} .
\end{aligned}
$$

We do not calculate the integrals in (5.12) for general $N$, but the 2 particle form factor follows from lemma 4 in appendix C.2

$$
F_{\alpha_{1} \alpha_{2}}^{\bar{\psi} \psi}(\underline{\theta})=\left\langle 0|\bar{\psi} \psi(0)| \theta_{1}, \theta_{2}\right\rangle_{\alpha_{1} \alpha_{2}}^{\text {in }}=\mathbf{C}_{\alpha_{1} \alpha_{2}} \bar{v}\left(\theta_{1}\right) u\left(\theta_{2}\right) F_{0}\left(\theta_{12}\right)
$$

and

$$
\begin{aligned}
F_{\alpha_{1} \alpha_{2}}^{T^{\mu \nu}}(\underline{\theta}) & =\left\langle 0\left|T^{\mu \nu}(0)\right| \theta_{1}, \theta_{2}\right\rangle_{\alpha_{1} \alpha_{2}}^{\text {in }}=\mathbf{C}_{\alpha_{1} \alpha_{2}} \bar{v}\left(\theta_{1}\right) \gamma^{\mu} u\left(\theta_{2}\right) \frac{1}{2}\left(p_{1}^{\nu}-p_{2}^{\nu}\right) F_{0}\left(\theta_{12}\right) \\
& =\mathbf{C}_{\alpha_{1} \alpha_{2}} \bar{v}\left(\theta_{1}\right) u\left(\theta_{2}\right) m \frac{\left(p_{1}-p_{2}\right)^{\mu}\left(p_{1}^{\nu}-p_{2}^{\nu}\right)}{\left(p_{1}-p_{2}\right)^{2}} F_{0}\left(\theta_{12}\right) \\
F_{\alpha_{1} \alpha_{2}}^{T}(\underline{\theta}) & =\left\langle 0|T(0)| p_{1}, p_{2}\right\rangle_{\alpha_{1} \alpha_{2}}^{\text {in }}=\mathbf{C}_{\alpha_{1} \alpha_{2}} \frac{\bar{v}\left(\theta_{1}\right) u\left(\theta_{2}\right)}{4 m \cosh ^{2} \frac{1}{2} \theta_{12}} F_{0}\left(\theta_{12}\right)
\end{aligned}
$$

with $F_{0}(\theta)$ given by $(3.9)$ and $(3.13)$.

1/N expansion. For $N \rightarrow \infty$ we obtain

$$
F_{\alpha_{1} \alpha_{2}}^{\bar{\psi} \psi}(\underline{\theta})=\mathbf{C}_{\alpha_{1} \alpha_{2}} \bar{v}\left(\theta_{2}\right) u\left(\theta_{1}\right) \frac{2 \operatorname{coth} \frac{1}{2} \theta_{12}}{\theta_{12}-i \pi}+O(1 / N)
$$

This result agrees with the one obtained by computing Feynman graphs as was done in [6].

\section{Conclusions}

In this article we have enlarged our $O(N)$ Bethe Ansatz knowledge of the $O(N)$ GrossNeveu model, which exhibits a very rich bound state structure and, consequently, creates a rich form factor hierarchy. We have computed the form factors for the fundamental Fermi field, which transforms as a vector representation of $O(N)$. Then we have also constructed the form factors for the Noether current and the energy-momentum tensor. In addition for the two particle case we have proved the recursion relation for the higher level Kfunctions. Finally we have checked our results against the usual $1 / N$ expansion and found full agreement. In a forthcoming paper we will investigate the kink form factors, possibly proving a kink field equation. Moreover, we will perform a detailed analysis of the $O(6)$ Gross-Neveu model, a starting point in the nesting procedure. 


\section{Acknowledgments}

The authors have profited from discussions with A. Fring, R. Schrader and B. Schroer. H.B. thanks A. Belavin, A. Ferraz, V. Korepin, P. Sodano and P. Wiegmann for valuable discussions. H.B. acknowledges financial support from the Armenian grant 11-1c_028 and the Armenian-Russian grant AR-17. He is also grateful to the International Institute of Physics of UFRN (Natal) for hospitality. A.F. acknowledges financial support from CNPq (Conselho Nacional de Desenvolvimento Cientifico e Tecnologico). M.K. thanks J. Balog and P. Weisz for discussions and hospitality at the Max-Planck Institut für Physik (München), where parts of this work have been performed.

\section{A Proof of the main theorem 1}

The identity

$$
\int_{\mathcal{C}_{a}} d z \Gamma(a-z) f(z)=2 \pi i \operatorname{Res}_{z=a} \sum_{l=-\infty}^{\infty} \Gamma(a-z-l) f(z+l)
$$

where the $\mathcal{C}_{a}$ encircles the poles of $\Gamma(a-z)$ anti-clockwise may be used to write the Kfunction $K_{\underline{\alpha}}^{\mathcal{O}}(\underline{\theta})$ defined by the integral representation (1.4) as a sum of "Jackson-type Integrals" as investigated in [1]. These expressions satisfy symmetry properties and a matrix difference equation which are equivalent to the form factor equations (i) and (ii). We have to prove, that due to the assumptions of theorem 1 in addition the residue relations (iii)

$$
\underset{\theta_{12}=i \pi}{\operatorname{Res}} F_{1 \ldots n}^{\mathcal{O}}\left(\theta_{1}, \ldots, \theta_{n}\right)=2 i \mathbf{C}_{12} F_{3 \ldots n}^{\mathcal{O}}\left(\theta_{3}, \ldots, \theta_{n}\right)\left(\mathbf{1}-\sigma_{2}^{\mathcal{O}}(\sigma S)_{2 n} \ldots(\sigma S)_{23}\right)
$$

and (iv)

$$
\underset{\theta_{12}=i \pi \nu}{\operatorname{Res}} F_{12 \ldots n}^{\mathcal{O}}\left(\theta_{1}, \theta_{2}, \ldots, \theta_{n}\right)=F_{(12) \ldots n}^{\mathcal{O}}\left(\theta_{(12)}, \ldots, \theta_{n}\right) \sqrt{2} \Gamma_{12}^{(12)}
$$

are satisfied.

Proof. We prove that the K-function $K_{1 \ldots n}^{\mathcal{O}}(\underline{\theta})$ defined by (1.3) and (1.4) satisfies the form factor equations (i)-(iii) which read in terms of $K_{1 \ldots n}^{\mathcal{O}}(\underline{\theta})$ as

$$
\begin{gathered}
K_{\ldots i j \ldots}^{\mathcal{O}}\left(\ldots, \theta_{i}, \theta_{j}, \ldots\right)=K_{\ldots j i \ldots}^{\mathcal{O}}\left(\ldots, \theta_{j}, \theta_{i}, \ldots\right) \tilde{S}_{i j}\left(\theta_{i j}\right) \\
K_{1 \ldots n}^{\mathcal{O}}\left(\theta_{1}+2 \pi i, \theta_{2}, \ldots, \theta_{n}\right) \sigma_{1}^{\mathcal{O}} \mathbf{C}^{\overline{1} 1}=K_{2 \ldots n 1}^{\mathcal{O}}\left(\theta_{2}, \ldots, \theta_{n}, \theta_{1}\right) \mathbf{C}^{1 \overline{1}} \\
\operatorname{Res}_{\theta_{12}=i \pi} K_{1 \ldots n}(\underline{\theta})=\frac{2 i}{F(i \pi)} \mathbf{C}_{12} \prod_{i=3}^{n} \tilde{\phi}\left(\theta_{i 1}+i \pi \nu\right) \tilde{\phi}\left(\theta_{i 2}\right) K_{3 \ldots n}\left(\theta_{3}, \ldots, \theta_{n}\right)\left(\mathbf{1}-\sigma_{2}^{\mathcal{O}} S_{2 n} \ldots S_{23}\right)
\end{gathered}
$$

where (4.9) has been used.

- (i) follows as in [1],

- (ii) follows as in [1], however, here (4.10) is responsible for the statistics factor $\sigma_{1}^{\mathcal{O}}$ in (A.3). 
- (iii) the residue of

$$
K_{1 \ldots n}^{\mathcal{O}}(\underline{\theta})=N_{n}^{\mathcal{O}} \int_{\mathcal{C}_{\underline{\theta}}^{(1)}} d z_{1} \cdots \int_{\mathcal{C}_{\underline{\theta}}^{(m)}} d z_{m} \tilde{h}(\underline{\theta}, \underline{z}) p^{\mathcal{O}}(\underline{\theta}, \underline{z}) \tilde{\Psi}_{1 \ldots n}(\underline{\theta}, \underline{z})
$$

consists of two terms

$$
\underset{\theta_{12}=i \pi}{\operatorname{Res}} K_{1 \ldots n}(\underline{\theta})=\left(\underset{\theta_{12}=i \pi}{\stackrel{(1)}{\operatorname{Res}}}+\underset{\theta_{12}=i \pi}{\operatorname{Res}}\right) K_{1 \ldots n}(\underline{\theta}) .
$$

This is because for each $z_{j}$ integration with $j$ even the contours will be "pinched" at two points (see figure 3 ):

(1) $z_{j}=\theta_{2} \approx \theta_{1}-i \pi$

(2) $z_{j}=\theta_{1}-2 \pi i \approx \theta_{2}-i \pi$

We prove in appendix C.1 the residue formulas for general level $k$ of the off-shell Bethe Ansatz. In particular for $k=0$ the general result implies that the pinching (1) gives

$$
\underset{\theta_{12}=i \pi}{\stackrel{(1)}{R e s}} K_{1 \ldots n}(\underline{\theta})=\frac{2 i}{F(i \pi)} \mathbf{C}_{12} \prod_{i=3}^{n} \tilde{\phi}\left(\theta_{i 1}+i \pi \nu\right) \tilde{\phi}\left(\theta_{i 2}\right) K_{3 \ldots n}\left(\theta_{3}, \ldots, \theta_{n}\right)
$$

for a suitable choice of the normalization constants in (A.5). Therefore we have proved

$$
\underset{\theta_{12}=i \pi}{\stackrel{(1)}{R e s}} F_{1 \ldots n}\left(\theta_{1}, \ldots, \theta_{n}\right)=2 i \mathbf{C}_{12} F_{3 \ldots n}\left(\theta_{3}, \ldots, \theta_{n}\right) .
$$

We use (ii) and (i) to write

$$
\begin{aligned}
F_{1 \ldots n}(\underline{\theta}) \sigma_{1}^{\mathcal{O}} & =\mathbf{C}_{1 \overline{1}} F_{2 \ldots n 1}\left(\theta_{2}, \ldots, \theta_{n}, \theta_{1}-2 \pi i\right) \mathbf{C}^{1 \overline{1}} \\
& =\mathbf{C}_{1 \overline{1}} F_{21 \ldots n}\left(\theta_{2}, \theta_{1}-2 \pi i, \ldots, \theta_{n}\right) \mathbf{C}^{1 \overline{1}}(\sigma S)_{\overline{1} n} \ldots(\sigma S)_{\overline{1} 3} .
\end{aligned}
$$

Then the result for $\underset{\theta_{1}=\theta_{2}+i \pi}{\stackrel{(1)}{R e s}}$ implies for the contribution of the pinching at $z_{j}=\theta_{1}-2 \pi i \approx$ $\theta_{2}-i \pi$

$$
\begin{aligned}
\underset{\theta_{1}=\theta_{2}+i \pi}{\operatorname{Res}} F_{1 \ldots n}(\underline{\theta}) \sigma_{1}^{\mathcal{O}} & =-\underset{\theta_{2}=\left(\theta_{1}-2 \pi i\right)+i \pi}{\operatorname{Res}} \mathbf{C}_{1 \overline{1}} F_{21 \ldots n}\left(\theta_{2}, \theta_{1}-2 \pi i, \ldots, \theta_{n}\right) \mathbf{C}^{1 \overline{1}}(\sigma S)_{\overline{1} n} \ldots(\sigma S)_{\overline{1} 3} \\
& =-\mathbf{C}_{1 \overline{1}} 2 i \mathbf{C}_{21} F_{3 \ldots n}^{\mathcal{O}}\left(\theta_{3}, \ldots, \theta_{n}\right) \mathbf{C}^{1 \overline{1}}(\sigma S)_{\overline{1} n} \ldots(\sigma S)_{\overline{1} 3} \\
& =-2 i \mathbf{C}_{12} F_{3 \ldots n}^{\mathcal{O}}\left(\theta_{3}, \ldots, \theta_{n}\right) \sigma_{2}^{\mathcal{O}}(\sigma S)_{2 n} \ldots(\sigma S)_{23} \sigma_{1}^{\mathcal{O}}
\end{aligned}
$$

using $\sigma_{1}^{\mathcal{O}} \sigma_{\overline{1}}^{\mathcal{O}}=1$.

(iv) Because there are bound states we also have to discuss the form factor equation (iv) $(3.4)$

$$
\underset{\theta_{12}=i \pi \nu}{\operatorname{Res}} F_{12 \ldots n}^{\mathcal{O}}\left(\theta_{1}, \theta_{2}, \underline{\hat{\theta}}\right)=F_{(12) \ldots n}^{\mathcal{O}}\left(\theta_{(12)}, \underline{\hat{\theta}}\right) \sqrt{2} \Gamma_{12}^{(12)}
$$


The bound state form factor $F_{(12) \ldots n}^{\mathcal{O}}\left(\theta_{(12)}, \underline{\hat{\theta}}\right)$ is then obtained from the residue

$$
\underset{\theta_{12}=i \pi \nu}{\operatorname{Res}} K_{12 \ldots n}^{\mathcal{O}}(\underline{\theta})=\operatorname{Res}_{\theta_{12}=i \pi \nu} N_{n}^{\mathcal{O}} \int_{\mathcal{C}_{\underline{\theta}}^{(1)}} d z_{1} \cdots \int_{\mathcal{C}_{\underline{\theta}}^{(m)}} d z_{m} \tilde{h}(\underline{\theta}, \underline{z}) p^{\mathcal{O}}(\underline{\theta}, \underline{z}) \tilde{\Psi}_{1 \ldots n}(\underline{\theta}, \underline{z}) .
$$

Similar as in the proof of (iii) the residue is obtained from pinching at:

$$
z_{j}=\theta_{1}-i \pi \nu \approx \theta_{2} \text { for } \mathcal{C}^{(o)} \text { and } z_{j}=\theta_{2} \approx \theta_{1}-i \pi \nu \text { for } \mathcal{C}^{(e)}
$$

Here we will not perform the lengthy calculations and write the complicated result, but in appendix D we will calculate the bound state form factors for the examples of section 5 .

\section{B Two-particle current form factor}

Derivation of (5.3) and (5.4):

Proof. The two-particle K-function of the current is

$$
K_{\underline{\alpha}}^{J}(\underline{\theta})=N_{2}^{J} \int_{\mathcal{C}_{\underline{\theta}}^{(o)}} d z \tilde{h}(\underline{\theta}, \underline{z}) p^{J}(\underline{\theta}, z) \tilde{\Psi}_{\underline{\alpha}}(\underline{\theta}, z)
$$

with the p-function (5.2) for $n=2$ and $m=1$

$$
p^{J}(\underline{\theta}, \underline{z})=\frac{e^{\frac{1}{2}\left(\theta_{1}+\theta_{2}\right)}}{e^{\theta_{1}}+e^{\theta_{2}}}+\frac{e^{-\frac{1}{2}\left(\theta_{1}+\theta_{2}\right)}}{e^{-\theta_{1}}+e^{-\theta_{2}}}=\frac{1}{\cosh \frac{1}{2} \theta_{12}}
$$

and the Bethe state

$$
\tilde{\Psi}_{\underline{\alpha}}(\underline{\theta}, z)=\delta_{\alpha_{1}}^{2} \delta_{\alpha_{2}}^{1} \tilde{c}\left(\theta_{1}-z\right)+\delta_{\alpha_{1}}^{1} \delta_{\alpha_{2}}^{2} \tilde{b}\left(\theta_{1}-z\right) \tilde{c}\left(\theta_{2}-z\right) .
$$

Doing the integral we obtain

$$
K_{21}^{J}\left(\theta_{12}\right)=N_{2}^{J} \int_{\mathcal{C}_{\underline{\theta}}^{(o)}} d z \tilde{h}(\underline{\theta}, z) p^{J}(\underline{\theta}, z) \tilde{\Psi}_{21}(\underline{\theta}, z)=-N_{2}^{J} 8 \pi^{3} 4^{\nu} \Gamma(1-\nu) c \frac{F_{-}(\theta)}{F(\theta)}
$$

and $K_{12}^{J}(\theta)=-K_{21}^{J}(\theta)$.

We use again the variables $u=\theta /(i \pi \nu)$ and $v=z /(i \pi \nu)$, consider the component $K_{21}^{J}(\theta)$ and calculate the integral

$$
\begin{aligned}
I & =\frac{1}{2 \pi i} \int_{\mathcal{C}_{\underline{u}}^{(o)}} d v I(\underline{u}, v) \\
I(\underline{u}, v) & =\tilde{h}(\underline{u}, v) \tilde{\Psi}(\underline{u}, v), \tilde{h}(\underline{u}, v)=\tilde{\phi}\left(u_{1}-v\right) \tilde{\phi}\left(u_{2}-v\right), \tilde{\Psi}(\underline{u}, v)=\tilde{c}\left(u_{1}-v\right) .
\end{aligned}
$$

Writing the integrals in terms of sums over residues we obtain (see figure 3 )

$$
\begin{array}{r}
I=I_{1}+I_{2}=\sum_{l=0}^{\infty} s_{1}\left(u_{1}, u_{2}, l\right)+\sum_{l=0}^{\infty} s_{2}\left(u_{1}, u_{2}, l\right) \\
s_{i}\left(u_{1}, u_{2}, l\right)=\operatorname{Res}_{v=v_{o}\left(u_{i}, l\right)} I\left(u_{1}, u_{2}, v\right), v_{o}(u, l)=u-1+2 l / \nu .
\end{array}
$$


Using the Gauss formula

$$
{ }_{2} F_{1}(a, b ; c ; 1)=\sum_{n=0}^{\infty} \frac{\Gamma(a+n)}{\Gamma(a)} \frac{\Gamma(b+n)}{\Gamma(b)} \frac{\Gamma(c)}{\Gamma(c+n)} \frac{\Gamma(1)}{\Gamma(1+n)}=\frac{\Gamma(c) \Gamma(c-a-b)}{\Gamma(c-a) \Gamma(c-b)}
$$

we get

$$
\begin{aligned}
I & =I_{1}+I_{2} \\
& =2^{\nu} \pi \sqrt{\pi} \frac{\Gamma\left(-\frac{1}{2} \nu\right) \Gamma\left(\frac{1}{2} \nu+\frac{1}{2}\right) \cos \frac{1}{2} \pi \nu}{\sin \frac{1}{2} \pi \nu\left(u_{12}+1\right) \sin \frac{1}{2} \pi \nu\left(u_{12}-1\right)} \frac{1}{\Gamma\left(\frac{1}{2} \nu+\frac{1}{2} \nu u_{12}\right) \Gamma\left(1+\frac{1}{2} \nu-\frac{1}{2} \nu u_{12}\right)}
\end{aligned}
$$

which agrees with (B.2) taking (B.1) into account. Therefore using (3.11) and (3.13) we finally obtain with the normalization constant

$$
N_{2}^{J}=\frac{1}{8} 4^{-\nu} \frac{i m}{c \pi^{3} \Gamma(1-\nu)}
$$

for the pseudo-potential $J^{\alpha \beta}(x)$ the two-particle form factors (5.3) and (5.4) for the current. The normalization is chosen such that the form factor agrees for $F_{-}(\theta) \rightarrow F_{-}(i \pi)=1$ with the free field expression.

\section{Higher level K-functions}

\section{C.1 Proof of lemma 2}

(1)

Remark 3 If in (4.15) Res is replaced by Res Lemma 2 also holds for $k=0$ as explained in appendix $A$.

For the discussion of the general $k$-level Bethe Ansatz it is convenient to use the variables $u, v$ defined by $\theta=i \pi \nu_{k} u, z=i \pi \nu_{k} v$ and $\nu_{k}=2 /(N-2 k-2)$ (for the S-matrix see $(4.11))$. In the proof we will replace $p^{(k)}(\underline{u}, \underline{v})$ by 1 which will not change the results, if the $p^{(k)}$ satisfy the conditions (4.17).

Proof. As above in the proof of theorem 1 the relations (i) ${ }^{(k)}$ and (ii) ${ }^{(k)}$ follow from the results of [1]. The proof of (iii) ${ }^{(k)}$ is the same as the corresponding one in [3], only the functions $\tilde{\psi}(u)$ and $\tilde{\chi}(u)$ have to replaced by $\tilde{\phi}(u)$ and $\tau_{i j}(v)$ by $\tau(v)$. As in [3] one finally obtains

$$
\begin{aligned}
\underset{u_{12}=1 / \nu_{k}}{\operatorname{Res}} K_{\underline{\alpha}}^{(k)}(\underline{u}) & \\
= & \text { const. }\left(\operatorname{Res}_{v=1 / \nu_{k+1}} \tilde{d}_{k+1}(v)\right)^{-1} \operatorname{Res}_{u_{12}=1 / \nu_{k}} \oint_{u_{1}-1} d v_{1} \tilde{c}\left(u_{1}-v_{1}\right)\left(-\oint_{u_{2}}\right) d v_{2} \\
& \times \tilde{d}_{k}\left(u_{12}\right)\left(\prod_{i=1}^{2} \prod_{j=1}^{2} \tilde{\phi}\left(u_{i}-v_{j}\right)\right) \tau\left(v_{12}\right) \prod_{i=3}^{n_{k}} \tilde{\phi}\left(u_{i 1}+1\right) \tilde{\phi}\left(u_{i 2}\right) \frac{1}{\tilde{N}_{m_{k}-2}^{(k)}} \mathbf{C}_{\alpha_{1} \alpha_{2}} K_{\underline{\alpha}}^{(k)}(\underline{\tilde{u}})
\end{aligned}
$$

with $\underline{\check{\alpha}}=\alpha_{3} \ldots \alpha_{n_{k}}$. It has been used that for $u_{12}=1 / \nu_{k}, v_{12}=1 / \nu_{k+1}, u_{2}=v_{2}, u_{1}=$ $v_{2}+1 / \nu_{k}=v_{1}+1$

$$
\left(\frac{a_{k+1}\left(v_{1 j}\right) a_{k+1}\left(v_{2 j}\right)}{a_{k}\left(u_{1}-v_{j}\right) a_{k}\left(u_{2}-v_{j}\right)} \tilde{\phi}\left(v_{j 1}+1\right) \tilde{\phi}\left(v_{j 2}\right)\right)\left(\tilde{\phi}\left(u_{1}-v_{j}\right) \tilde{\phi}\left(u_{2}-v_{j}\right) \tau\left(v_{1 j}\right) \tau\left(v_{2 j}\right)\right)=1 .
$$


This can be shown by means of (4.5) and the formulas

$$
\begin{aligned}
a_{k}\left(u_{1}\right) a_{k}\left(u_{2}\right) & =\tilde{b}\left(-u_{2}\right) / \tilde{b}\left(u_{1}\right) \\
\tilde{b}(u) \tilde{\phi}(u) & =-\tilde{\phi}(1-u) .
\end{aligned}
$$

The final result is that equation (4.15) holds for a suitable choice of the normalization constants in (4.12).

\section{C.2 Two-particle higher level K-functions}

We need higher level K-functions for the examples of section 5, in particular, in the isoscalar two-particle channel (with weights $w=(0, \ldots, 0))$ the K-function $K_{\alpha_{1} \alpha_{2}}^{(k)}\left(\theta_{1}, \theta_{2}\right)$ (level $k=0,1,2, \ldots)$ belonging to $O(N-2 k)$. It is of the form

$$
K_{\alpha_{1} \alpha_{2}}^{(k)}\left(u_{1}, u_{2}\right)=\mathbf{C}_{\alpha_{1} \alpha_{2}}^{(N-2 k)} K\left(u_{12}, k\right)
$$

where $\mathbf{C}_{\alpha_{1} \alpha_{2}}^{(N-2 k)}$ is the $O(N-2 k)$ charge conjugation matrix. ${ }^{10}$ From the weight vector

$$
w=\left(w_{1}, \ldots, w_{N / 2}\right)=(0, \ldots, 0)=\left(n-n_{1}, \ldots, n_{N / 2-2}-n_{-}-n_{+}, n_{-}-n_{+}\right)
$$

follows that for all levels $n_{k}=2$.

Lemma 4 The vector valued functions $K_{\alpha_{1} \alpha_{2}}^{(k)}\left(u_{1}, u_{2}\right)$ with

$$
K(u, k)=\frac{\Gamma\left(1-\frac{1}{2} \nu-\frac{1}{2} \nu u\right) \Gamma\left(-\frac{1}{2} \nu+\frac{1}{2} \nu u\right)}{\Gamma\left(\frac{3}{2}-\frac{1}{2} k \nu-\frac{1}{2} \nu u\right) \Gamma\left(\frac{1}{2}-\frac{1}{2} k \nu+\frac{1}{2} \nu u\right)}
$$

satisfy for $k=0,1,2, \cdots<N / 2-2$ the recursion relation

$$
\begin{aligned}
K_{\underline{\alpha}}^{(k)}(\underline{u}) & =N^{(k)} \int_{\mathcal{C}_{\underline{u}}^{(o)}} d v_{1} \int_{\mathcal{C}_{\underline{u}}^{(e)}} d v_{2} \tilde{h}(\underline{u}, \underline{v}) L_{\underline{\hat{\beta}}}^{(k)}(\underline{v}, k) \tilde{\Phi}^{(k)} \underline{\underline{\beta}} \underline{\underline{\alpha}}(\underline{u}, \underline{v}) \\
L_{\underline{\hat{\beta}}}^{(k)}(\underline{v}, k) & =K_{\underline{\underline{\beta}}}^{(k+1)}(\underline{v})=\mathbf{C}_{\underline{\underline{\beta}}}^{(N-2 k-2)} K\left(v_{12}, k+1\right)
\end{aligned}
$$

with

$$
\begin{aligned}
\tilde{h}(\underline{u}, \underline{v}) & =\prod_{i=1}^{2}\left(\tilde{\phi}\left(u_{i}-v_{1}\right) \tilde{\phi}\left(u_{i}-v_{2}\right)\right) \frac{1}{\tilde{\phi}\left(v_{12}\right) \tilde{\phi}\left(-v_{12}\right)} \\
\tilde{\Phi}^{(k)} \underline{\underline{\beta}}(\underline{\underline{u}}, \underline{v}) & =\left(\Pi \underline{\underline{\beta}}(\underline{v}) \Omega \tilde{T}_{1}^{\beta_{2}}\left(\underline{u}, v_{2}\right) \tilde{T}_{1}^{\beta_{1}}\left(\underline{u}, v_{1}\right)\right)_{\underline{\alpha}}^{(k)}
\end{aligned}
$$

and the normalization

$$
N^{(k)}=\frac{-2^{-\nu}}{8 \pi^{2}} \frac{\Gamma\left(\frac{1}{2}-\frac{1}{2} k \nu\right) \Gamma\left(1-\frac{1}{2} k \nu-\frac{1}{2} \nu\right)}{\Gamma\left(1-\frac{1}{2} k \nu\right) \Gamma\left(\frac{1}{2}-\frac{1}{2} k \nu+\frac{1}{2} \nu\right)\left(\Gamma\left(-\frac{1}{2} \nu\right)\right)^{2}} .
$$

\footnotetext{
${ }^{10}$ In the real basis this would be $\delta_{\alpha_{1} \alpha_{2}}$.
} 
Proof. The function (C.2) satisfies

$$
\begin{aligned}
\text { (i) : } & K(u, k) & =K(-u, k) \tilde{S}_{0}^{(k)}(u) \\
\text { (ii) }: & K(1 / \nu-u, k) & =K(1 / \nu+u, k)
\end{aligned}
$$

with the scalar eigenvalue of $\tilde{S}^{(k)}(u)=\tilde{S}^{O(N-2 k)}(u)$

$$
\tilde{S}_{0}^{(k)}(u)=S_{0}^{(k)}(u) / S_{+}^{(k)}(u)=\frac{u+1 / \nu_{k}}{u-1 / \nu_{k}} \frac{u+1}{u-1}=\frac{u+(1 / \nu-k)}{u-(1 / \nu-k)} \frac{u+1}{u-1} .
$$

The minimal solution (with no poles in the physical strip $0 \leq \operatorname{Re} u \leq 1 / \nu$ ) is

$$
K_{m}(u, k)=\frac{1}{\Gamma\left(\frac{3}{2}-\frac{1}{2} k \nu-\frac{1}{2} \nu u\right) \Gamma\left(\frac{1}{2}(1-\nu k)+\frac{1}{2} \nu u\right) \Gamma\left(1+\frac{1}{2} \nu-\frac{1}{2} \nu u\right) \Gamma\left(\frac{1}{2} \nu+\frac{1}{2} \nu u\right)}
$$

whereas $K(u, k)$ has the bound state pole at $u=1\left(\theta=i \pi \nu_{k}\right)$.

The Bethe state in (C.3) is (see [1])

$$
\begin{aligned}
\tilde{\Phi}^{(k)} \frac{\stackrel{\leftrightarrow}{\alpha}}{\underline{\alpha}}(\underline{u}, \underline{v}) & =\left(\Pi^{(k)}\right)_{\underline{\beta}}^{\underline{\beta}}(\underline{v})\left(\Omega \tilde{T}_{1}^{\beta_{2}}\left(\underline{u}, v_{2}\right) \tilde{T}_{1}^{\beta_{1}}\left(\underline{u}, v_{1}\right)\right)_{\underline{\alpha}}^{(k)} \\
\left(\Pi^{(k)}\right)_{\beta_{1} \beta_{2}}^{\stackrel{\leftrightarrow}{\beta}_{1} \dot{\beta}_{2}}(\underline{v}) & =\delta_{\beta_{1}}^{\dot{\beta}_{1}} \delta_{\beta_{2}}^{\dot{\beta}_{2}}+f_{k}\left(v_{12}\right){\stackrel{\mathbf{C}}{\dot{\beta}_{1} \dot{\beta}_{2}}}_{\delta_{\beta_{1}}^{k+1}} \delta_{\beta 2}^{k+1}, \quad f_{k}(v)=\frac{1}{v+1 / \nu_{k}-1}
\end{aligned}
$$

which may be depicted (for $k=0$ ) as

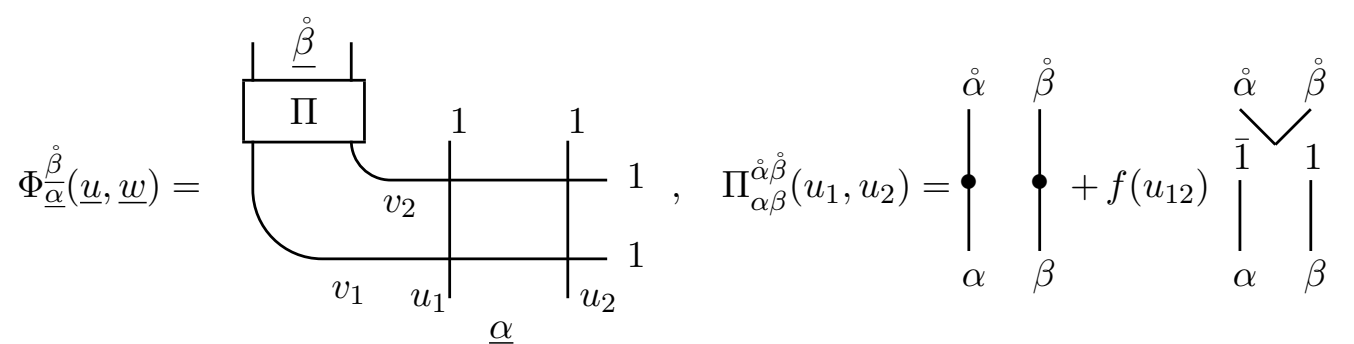

Because of (C.1) it is sufficient to consider only one component of (C.3) and for convenience we take $\tilde{\Phi}^{(k)} \underline{\underline{\beta}}$ with $\underline{\alpha}=\overline{k+1}, k+1$ and define

$$
\begin{aligned}
\tilde{\Phi}_{k}(\underline{u}, \underline{v}) \\
\quad=\mathbf{C}_{\dot{\beta}_{1} \dot{\beta}_{2}}^{(N-2 k-2)} \tilde{\Phi}^{(k)} \frac{\stackrel{\leftrightarrow}{\beta}_{1} \stackrel{\leftrightarrow}{2}_{2}}{k+1, k+1}(\underline{u}, \underline{v}) \\
\quad=\mathbf{C}_{\dot{\beta}_{1} \dot{\beta}_{2}}^{(N-2 k-2)} \mathbf{C}_{(N-2 k-2)}^{\dot{\beta}_{1} \stackrel{\beta}{2}_{2}}\left(\tilde{c}\left(u_{1}-v_{2}\right) \tilde{d}_{k}\left(u_{1}-v_{1}\right)+f_{k}\left(v_{1}-v_{2}\right)\left(\tilde{c}\left(u_{1}-v_{1}\right)+\tilde{d}_{k}\left(u_{1}-v_{1}\right)\right)\right) \\
\quad=(N-2 k-2) \frac{-\left(v_{1}-v_{2}-1\right)}{\left(u_{1}-v_{1}-1\right)\left(u_{1}-v_{2}-1\right)\left(v_{1}-v_{2}+1 / \nu_{k}-1\right)}
\end{aligned}
$$

where (for $k=0) \tilde{\Phi}_{\overline{1}, 1}^{\dot{\beta}_{1} \dot{\beta}_{2}}(\underline{u}, \underline{v})$ may be depicted as

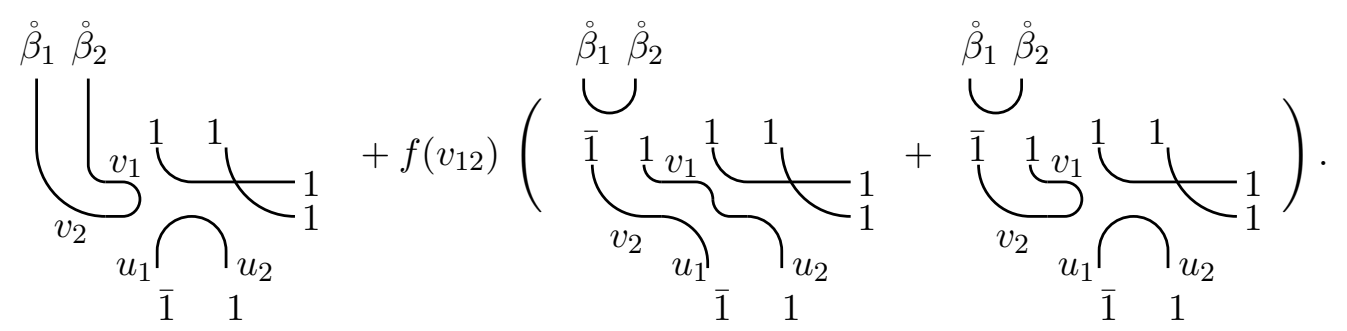


Then because $L_{\underline{\beta}}^{(k)}(\underline{v}) \tilde{\Phi}^{(k)} \frac{\stackrel{\circ}{\xi}}{\bar{k}+1, k+1}(\underline{u}, \underline{v})=K\left(v_{12}, k+1\right) \tilde{\Phi}_{k}(\underline{u}, \underline{v})$

$$
\begin{aligned}
K_{\overline{k+1, k+1}}^{(k)}(\underline{u}) & =N^{(k)} \int_{\mathcal{C}_{\underline{u}}^{(o)}} d v_{1} \int_{\mathcal{C}_{\underline{u}}^{(e)}} d v_{2} \tilde{h}(\underline{u}, \underline{v}) K\left(v_{12}, k+1\right) \tilde{\Phi}_{k}(\underline{u}, \underline{v}) \\
& =N^{(k)}(2 \pi i)^{2}(N-2 k-2) J\left(u_{12}\right)
\end{aligned}
$$

where

$$
\begin{aligned}
J\left(u_{12}\right) & =\frac{1}{(2 \pi i)^{2}} \int_{\mathcal{C}_{\underline{u}}^{(o)}} d v_{1} \int_{\mathcal{C}_{\underline{u}}^{(e)}} d v_{2} J(\underline{u}, \underline{v}) \\
J(\underline{u}, \underline{v}) & =\tilde{\phi}\left(u_{1}-v_{1}\right) \tilde{c}\left(u_{1}-v_{1}\right) \tilde{\phi}\left(u_{1}-v_{2}\right) \tilde{c}\left(u_{1}-v_{2}\right) \tilde{\phi}\left(u_{2}-v_{1}\right) \tilde{\phi}\left(u_{2}-v_{2}\right) \varphi\left(v_{12}\right) \\
\varphi(v) & =\frac{(1-v) K(v, k+1)}{\tilde{\phi}(v) \tilde{\phi}(-v)(v+1 / \nu-k-1)} .
\end{aligned}
$$

Because the function $J(u)$ satisfies (C.5) it is proportional to $K(u, k)$ (as was shown in general in [6]) if there are no zeroes and exactly one pole ${ }^{11}$ at $u=1$ in $0 \leq \operatorname{Re} u \leq 1 / \nu$. Finally we obtain

$$
J(u)=\frac{2^{\nu-1} \nu\left(\Gamma\left(-\frac{1}{2} \nu\right)\right)^{2} \Gamma\left(\frac{1}{2}-\frac{1}{2} k \nu+\frac{1}{2} \nu\right) \Gamma\left(1-\frac{1}{2} k \nu\right)}{\Gamma\left(-\frac{1}{2} \nu+1-\frac{1}{2} k \nu\right) \Gamma\left(\frac{3}{2}-\frac{1}{2} k \nu\right)} K(u, k)
$$

where the constant is calculated by taking the residue at $u=1$ on both sides of (C.10). Finally we turn to (C.3). By (C.8) and (C.1) we have

$$
K(u, k)=K_{k+1, k+1}^{(k)}(\underline{u})=N^{(k)}(2 \pi i)^{2}(N-2 k-2) J\left(u_{12}\right)
$$

which provides the normalization (C.4).

In particular for $k=0$ and $k=1$

$$
\begin{aligned}
& K(u)=K(u, 0)=\frac{-2}{\pi} \frac{\cos \frac{1}{2} \pi \nu u}{\nu u-1} \Gamma\left(-\frac{1}{2} \nu+\frac{1}{2} \nu u\right) \Gamma\left(1-\frac{1}{2} \nu-\frac{1}{2} \nu u\right) \\
& L(u)=K(u, 1)=\frac{\Gamma\left(1-\frac{1}{2} \nu-\frac{1}{2} \nu u\right) \Gamma\left(-\frac{1}{2} \nu+\frac{1}{2} \nu u\right)}{\Gamma\left(1+\frac{1}{2}(1-\nu)-\frac{1}{2} \nu u\right) \Gamma\left(\frac{1}{2}(1-\nu)+\frac{1}{2} \nu u\right)} .
\end{aligned}
$$

The function $L(u)$ is that of (5.10) and it is used to calculate the 2-particle form factor on the energy momentum (5.14) and also to calculate the 3-particle form factor of the field 5.9. In particular the 2-particle K-function of the scalar operator $\bar{\psi} \psi$ is up to a constant equal to $K(u)$. With the normalization in (5.12)

$$
N_{2}^{\bar{\psi} \psi}=2 m /\left(\pi^{2} \nu^{2} c \Gamma^{2}\left(\frac{1}{2}(1-\nu)\right)\right)
$$

we obtain (5.14)

$$
F_{\alpha_{1} \alpha_{2}}^{\bar{\psi} \psi}(\underline{\theta})=\mathbf{C}_{\alpha_{1} \alpha_{2}} \bar{v}\left(\theta_{1}\right) u\left(\theta_{2}\right) F_{0}\left(\theta_{12}\right)
$$

which agrees with the result of [6]. The normalization is chosen such that the form factor agrees for $\theta \rightarrow i \pi$ with the free field expression.

\footnotetext{
${ }^{11}$ This is suggested by numerical calculations using mathematica.
} 


\section{Bound state form factors}

We discuss the form factor equation (iv)

$$
\underset{\theta_{12}=i \eta}{\operatorname{Res}} F_{12 \ldots n}^{\mathcal{O}}\left(\theta_{1}, \theta_{2}, \ldots, \theta_{n}\right)=F_{(12) \ldots n}^{\mathcal{O}}\left(\theta_{(12)}, \ldots, \theta_{n}\right) \sqrt{2} \Gamma_{12}^{(12)}
$$

for the examples of section (5). Of course, one may easily calculate the residues for twoparticle form factors for the pseudo-potential $J^{\alpha \beta}(x)(5.3)$ and $\bar{\psi} \psi(x)(5.14)$ directly, however we will check here whether the general pinching procedure of appendix A will give the same result. In addition we obtain the bound state form factor of the three-particle form factor for the field.

Two-particle current form factor. By the form factor equation (iv) (3.4) the twoparticle bound state form factor for the pseudo-potential $J^{\alpha \beta}(x)$ is

$$
\underset{\theta_{12}=i \pi \nu}{\operatorname{Res}} F_{12}^{J^{\alpha \beta}}\left(\theta_{1}, \theta_{2}\right)=F_{(12)}^{J^{\alpha \beta}}\left(\theta_{(12)}\right) \sqrt{2} \Gamma_{12}^{(12)}, \quad \theta_{(12)}=\frac{1}{2}\left(\theta_{1}+\theta_{2}\right)
$$

where the bound state intertwiner $\Gamma_{12}^{(12)}$ is given by (2.9) and (2.10).

In appendix B we calculated the two-particle form factor for the pseudo-potential $J^{\alpha \beta}(x)$ in terms of the integral

$$
\begin{aligned}
I\left(u_{12}\right) & =\frac{1}{2 \pi i} \int_{\mathcal{C}_{\underline{u}}^{(o)}} d v I(\underline{u}, \underline{v}) \\
I(\underline{u}, v) & =\tilde{h}(\underline{u}, v) \tilde{\Psi}(\underline{u}, v), \tilde{h}(\underline{u}, v)=\tilde{\phi}\left(u_{1}-v\right) \tilde{\phi}\left(u_{2}-v\right), \tilde{\Psi}(\underline{u}, v)=\tilde{c}\left(u_{1}-v\right)
\end{aligned}
$$

with the result

$$
I(u)=\frac{2^{\nu} \pi \sqrt{\pi} \Gamma\left(-\frac{1}{2} \nu\right) \Gamma\left(\frac{1}{2} \nu+\frac{1}{2}\right) \cos \frac{1}{2} \pi \nu}{\sin \frac{1}{2} \pi \nu(u+1) \sin \frac{1}{2} \pi \nu(u-1) \Gamma\left(\frac{1}{2} \nu+\frac{1}{2} \nu u\right) \Gamma\left(1+\frac{1}{2} \nu-\frac{1}{2} \nu u\right)}
$$

and the residue

$$
\operatorname{Res}_{u=1} I(u)=-\left(\Gamma\left(-\frac{1}{2} \nu\right)\right)^{2}
$$

In appendix $\mathrm{A}$ we remarked that the residue is obtained from pinching at: $z=\theta_{1}-i \pi \nu \approx \theta_{2}$ for $\mathcal{C}^{(o)}$

$$
\begin{aligned}
\operatorname{Res}_{u_{12}=1} I\left(u_{12}\right) & =\operatorname{Res}_{u_{12}=1} \frac{1}{2 \pi i} \oint_{u_{1}-1} d v \tilde{\phi}\left(u_{1}-v\right) \tilde{\phi}\left(u_{2}-v\right) \tilde{c}\left(u_{1}-v\right) \\
& =\operatorname{Res}_{u_{12}=1} \tilde{\phi}(1) \tilde{\phi}\left(-u_{12}+1\right)=-\left(\Gamma\left(-\frac{1}{2} \nu\right)\right)^{2}
\end{aligned}
$$

which means that the pinching procedure gives the same result as the direct calculation.

Two-particle form factor of $\overline{\boldsymbol{\psi}} \boldsymbol{\psi}$. By the form factor equation (iv) (3.4) the twoparticle bound state form factor for $\bar{\psi} \psi$ is

$$
\underset{\theta_{12}=i \pi \nu}{\operatorname{Res}} F_{12}^{\bar{\psi} \psi}\left(\theta_{1}, \theta_{2}\right)=F_{(12)}^{\bar{\psi} \psi}\left(\theta_{(12)}\right) \sqrt{2} \Gamma_{12}^{(12)} .
$$


In appendix C.2 we calculated the two-particle form factor for $\bar{\psi} \psi(x)$ in terms of the integral

$$
\begin{aligned}
J\left(u_{12}\right) & =\frac{1}{(2 \pi i)^{2}} \int_{\mathcal{C}_{\underline{u}}^{(o)}} d v_{1} \int_{\mathcal{C}_{\underline{u}}^{(e)}} d v_{2} J(\underline{u}, \underline{v}) \\
J(\underline{u}, \underline{v}) & =\tilde{\phi}\left(u_{1}-v_{1}\right) \tilde{c}\left(u_{1}-v_{1}\right) \tilde{\phi}\left(u_{1}-v_{2}\right) \tilde{c}\left(u_{1}-v_{2}\right) \tilde{\phi}\left(u_{2}-v_{1}\right) \tilde{\phi}\left(u_{2}-v_{2}\right) \varphi\left(v_{12}\right) \\
\varphi(v) & =\frac{(1-v) K(v, k+1)}{\tilde{\phi}(v) \tilde{\phi}(-v)(v+1 / \nu-k-1)}
\end{aligned}
$$

with the result

$$
J=\frac{c_{2}}{\cos \pi \nu} K(u, 0)=\frac{c_{2}}{\cos \pi \nu} \frac{\Gamma\left(1-\frac{1}{2} \nu-\frac{1}{2} \nu u\right) \Gamma\left(-\frac{1}{2} \nu+\frac{1}{2} \nu u\right)}{\Gamma\left(1+\frac{1}{2}-\frac{1}{2} \nu u\right) \Gamma\left(\frac{1}{2}+\frac{1}{2} \nu u\right)}
$$

and the residue is

$$
\operatorname{Res}_{u=1} J(u)=\frac{c_{2}}{\cos \pi \nu} \operatorname{Res}_{u=1} K(u, 0)=\frac{4}{(1-\nu) \pi}\left(\Gamma\left(-\frac{1}{2} \nu\right)\right)^{2} .
$$

In appendix $\mathrm{A}$ we remarked that the residue is obtained from pinching at: $z=\theta_{1}-i \pi \nu \approx \theta_{2}$ for $\mathcal{C}^{(o)}$ and $z_{j}=\theta_{2} \approx \theta_{1}-i \pi \nu$ for $\mathcal{C}^{(e)}$, therefore (see (C.9))

$$
\begin{aligned}
\underset{u_{12}=1}{\operatorname{Res}} J & =\operatorname{Res}_{u_{12}=1} \frac{1}{(2 \pi i)^{2}}\left(\oint_{u_{1}-1} d v_{1} \int_{\mathcal{C}_{\underline{u}}^{(e)}} d v_{2}-\int_{\mathcal{C}_{\underline{u}}^{(o)}} d v_{1} \oint_{u_{2}} d v_{2}\right) J(\underline{u}, \underline{v})=R_{1}+R_{2} \\
R_{1} & =-\operatorname{Res}_{u_{12}=1} \sum_{l_{2}=0}^{\infty}\left(s_{11}\left(u_{1}, u_{2}, 0, l_{2}\right)+s_{12}\left(u_{1}, u_{2}, 0, l_{2}\right)\right) .
\end{aligned}
$$

with

$$
\begin{aligned}
s_{i j}\left(u_{1}, u_{2}, l_{1}, l_{2}\right) & =\operatorname{Res}_{v_{1}=v_{o}\left(u_{i}, l_{1}\right)} \underset{v_{2}=v_{e}\left(u_{j}, l_{2}\right)}{\operatorname{Res}} J\left(u_{1}, u_{2} ; v_{1}, v_{2}\right) \\
v_{o}(u, l) & =u-1+2 l / \nu, \quad v_{e}(u, l)=u-2 l / \nu
\end{aligned}
$$

It turns out that $s_{12}$ gives no contribution and

$$
R_{1}=-\operatorname{Res}_{u_{12}=1} \sum_{l_{2}=0}^{\infty} s_{11}\left(u_{1}, u_{2}, 0, l_{2}\right)=2 \frac{\left(\Gamma\left(-\frac{1}{2} \nu\right)\right)^{2}}{\pi(1-\nu)}
$$

such that again

$$
\operatorname{Res}_{u_{12}=1} J=4 \frac{\left(\Gamma\left(-\frac{1}{2} \nu\right)\right)^{2}}{\pi(1-\nu)}
$$

which means that the pinching procedure gives the same result as the direct calculation.

3-particle form factor of $\boldsymbol{\psi}$. We discuss the bound state fusion of 2 fundamental fermions $f+f \rightarrow b_{2}$. We write (5.5) as

$$
\psi(x)=(i \gamma \partial+m) \tilde{\chi}(x), \quad \tilde{\chi}(x)=-i\left(\square+m^{2}\right)^{-1} \chi(x)
$$


and apply the form factor equation (3.4) to $\tilde{\chi}^{12}$

$$
\underset{\theta_{12}=i \pi \nu}{\operatorname{Res}} F_{1 \overline{1} 1}^{\tilde{\chi}}(\underline{\theta})=F_{b_{2} 1}^{\tilde{\chi}^{( \pm)}}\left(\theta_{0}, \theta_{3}\right) \sqrt{2} \Gamma_{1 \overline{1}}^{b_{2}} .
$$

The component $K_{1 \overline{1} 1}^{\tilde{\chi}}$ of the K-function (similar as for $\bar{\psi} \psi$ in appendix C.2) can be written in terms of

$$
\begin{aligned}
J^{\chi}(\underline{u}) & =\frac{1}{(2 \pi i)^{2}} \int_{\mathcal{C}_{\underline{u}}^{(o)}} d v_{1} \int_{\mathcal{C}_{\underline{u}}^{(e)}} d v_{2} J^{\chi}(\underline{u}, \underline{v}) p^{\chi}(\underline{u}, \underline{v}) \\
J^{\chi}(\underline{u}, \underline{v}) & =\left(\prod_{i=1}^{3} \prod_{j=1}^{2} \tilde{\phi}\left(u_{i}-v_{j}\right)\right) \tilde{b}\left(u_{1}-v_{1}\right) \tilde{b}\left(u_{1}-v_{2}\right) \tilde{c}\left(u_{2}-v_{1}\right) \tilde{c}\left(u_{2}-v_{2}\right) \varphi\left(v_{12}\right) \\
\varphi(v) & =\frac{(1-v) K(v, 1)}{\tilde{\phi}(v) \tilde{\phi}(-v)(v+1 / \nu-1)} .
\end{aligned}
$$

In appendix $\mathrm{A}$ we remarked that the residue is obtained from pinching at:

$z_{1}=\theta_{1}-i \pi \nu \approx \theta_{2}\left(v_{1}=u_{1}-1 \approx u_{2}\right)$ for $\mathcal{C}^{(o)}$ and $z_{2}=\theta_{2} \approx \theta_{1}-i \pi \nu\left(v_{2}=u_{2} \approx u_{1}-1\right)$ for $\mathcal{C}^{(e)}$. Therefore the bound state form factor is obtained from

$$
\operatorname{Res}_{u_{12}=1} J^{\chi}(\underline{u})=\operatorname{Res}_{u_{12}=1} \frac{1}{(2 \pi i)^{2}}\left(\oint_{u_{1}-1} \int_{\mathcal{C}_{\underline{u}}^{(e)}}-\int_{\mathcal{C}_{\underline{u}}^{(o)}} \oint_{u_{2}}\right) d v_{1} d v_{2} J^{\chi}(\underline{u}, \underline{v}) p^{\chi}(\underline{u}, \underline{v}) .
$$

The integrals may be calculated in terms of hypergeometric functions ${ }_{3} F_{2}$. We obtain

$$
\begin{aligned}
F_{b_{2} 1}^{\tilde{\chi}^{( \pm)}}\left(\theta_{0}, \theta_{3}\right)= & e^{\mp \frac{1}{2} \theta_{0}}\left(e^{ \pm \frac{1}{4} i \pi \nu} f_{13}\left(\theta_{03}\right)+e^{\mp \frac{1}{4} i \pi \nu} f_{32}\left(\theta_{03}\right)\right) \\
& +e^{\mp \frac{1}{2} \theta_{3}}\left(e^{ \pm \frac{1}{2} i \pi \nu} f_{11}\left(\theta_{03}\right)+e^{\mp \frac{1}{2} i \pi \nu} f_{22}\left(\theta_{03}\right)\right)
\end{aligned}
$$

where $f_{1 i}\left(\theta_{03}\right)$ and $f_{i 2}\left(\theta_{03}\right)$ are the results from the integrations

$$
\oint_{u_{1}-1} d v_{1} \int_{\mathcal{C}_{u_{i}}} d v_{2} \ldots \text { and } \oint_{\mathcal{C}_{u_{i}}} d v_{1} \int_{u_{2}} d v_{2} \ldots \text { respectively. }
$$

For example up to a constant (see figure 4)

$$
\begin{aligned}
f_{13}(u)= & \frac{\left(\Gamma\left(1-\frac{1}{4} \nu-\frac{1}{2} \nu u\right)\right)^{2} \Gamma\left(-\frac{3}{4} \nu+\frac{1}{2} \nu u\right) \Gamma\left(-\frac{1}{4} \nu+\frac{1}{2} \nu u\right)}{\Gamma\left(\frac{3}{2}-\frac{3}{4} \nu+\frac{1}{2} \nu u\right) \Gamma\left(\frac{3}{2}-\frac{1}{4} \nu-\frac{1}{2} \nu u\right) \cot \frac{1}{2} \pi \nu\left(u-\frac{1}{2}\right) \cot \frac{1}{2} \pi \nu\left(u+\frac{1}{2}\right)} \\
& \times{ }_{3} F_{2}\left(-\frac{1}{2} \nu+1,-\frac{3}{4} \nu+\frac{1}{2} \nu u,-\frac{1}{2}+\frac{1}{4} \nu+\frac{1}{2} \nu u ; \frac{1}{4} \nu+\frac{1}{2} \nu u, \frac{3}{2}-\frac{3}{4} \nu+\frac{1}{2} \nu u ; 1\right) F_{b}(u)
\end{aligned}
$$

where $F_{b}(u)$ is the minimal highest weight form factor function in the $b_{2}^{(r)}+f$ sector

$$
F_{b}(\theta)=\text { const. }\left(\sinh \frac{1}{2} \theta\right) \frac{F_{+}^{\min }\left(\theta+\frac{1}{2} i \pi \nu\right) F_{+}^{\min }\left(\theta-\frac{1}{2} i \pi \nu\right)}{\Gamma\left(1+\frac{1}{4} \nu-\frac{\theta}{2 i \pi}\right) \Gamma\left(\frac{1}{4} \nu+\frac{\theta}{2 i \pi}\right)}
$$

or explicitly in terms of $G(z)$ Barnes G-function

$$
F_{b}(u)=\frac{\left(\sinh \frac{1}{2} \theta\right) G\left(\frac{1}{4} \nu+\frac{1}{2} \nu u\right) G\left(\frac{3}{2}-\frac{3}{4} \nu-\frac{1}{2} \nu u\right) G\left(1+\frac{1}{4} \nu-\frac{1}{2} \nu u\right) G\left(\frac{1}{2}-\frac{3}{4} \nu+\frac{1}{2} \nu u\right)}{G\left(\frac{1}{2}+\frac{1}{4} \nu+\frac{1}{2} \nu u\right) G\left(2-\frac{3}{4} \nu-\frac{1}{2} \nu u\right) G\left(\frac{3}{2}+\frac{1}{4} \nu-\frac{1}{2} \nu u\right) G\left(1-\frac{3}{4} \nu+\frac{1}{2} \nu u\right)}
$$

\footnotetext{
${ }^{12}$ Strictly speaking $F_{1 \overline{1} 1}^{\tilde{\chi}} \pm F_{111}^{\tilde{\chi}}$ give $F_{b_{2}^{(0,2)}}^{\tilde{\chi}}$.
} 
with $u=\theta /(i \pi \nu)$. It satisfies Watson's equation

$$
\frac{F_{b}(\theta)}{F_{b}(-\theta)}=a\left(\theta+\frac{1}{2} i \pi \nu\right) a\left(\theta-\frac{1}{2} i \pi \nu\right) \frac{\theta+\frac{1}{2} i \pi \nu}{\theta-\frac{1}{2} i \pi \nu}=a_{b}(\theta)
$$

where $a_{b}(\theta)$ is the highest weight scattering amplitude in the $b_{2}^{(r)}+f$ sector.

\section{E $1 / \mathrm{N}$ expansion}

\section{E.1 1/N expansion of the exact 3-particle field form factor}

For $\chi^{\delta}(x)=i(-i \gamma \partial+m) \psi^{\delta}(x)$ we derive for the highest weight component $\chi(x)=\chi^{1}(x)$

$$
F_{\overline{1} 11}^{\chi}(\underline{\theta})=\frac{8 \pi m}{N}\left(\frac{\cosh \frac{1}{2} \theta_{12}}{\theta_{12}-i \pi} u\left(\theta_{3}\right)-\frac{\cosh \frac{1}{2} \theta_{13}}{\theta_{13}-i \pi} u\left(\theta_{2}\right)\right)+O\left(N^{-2}\right)
$$

which is equivalent to (5.11).

Proof. The p-function of $\chi(x)$ for three particles and $\nu=0$ is

$$
p^{\chi^{( \pm)}}=\exp \left(\mp \frac{1}{2}\left(\theta_{1}+\theta_{2}+\theta_{3}-z_{1}-z_{2}\right)\right) .
$$

We have to consider (up to const.)

$$
K_{\overline{1} 11}^{\chi^{(+)}}(\underline{\theta})=\int_{\mathcal{C}_{\underline{\theta}}} d z_{1} \int_{\mathcal{C}_{\underline{\theta}}} d z_{2} \prod_{i=1}^{3}\left(\tilde{\phi}\left(\theta_{i}-z_{1}\right) \tilde{\phi}\left(\theta_{i}-z_{2}\right)\right) \frac{1}{\tilde{\phi}\left(z_{12}\right) \tilde{\phi}\left(-z_{12}\right)} p^{\chi^{(+)}}(\underline{z}) \tilde{\Psi}_{\overline{1} 11}(\underline{\theta}, \underline{z}) .
$$

This formula is similar as (C.3) for $k=0$ (which correspond to the operator $\bar{\psi} \psi$ ), only we have here to add the factor $\left(\tilde{\phi}\left(\theta_{3}-z_{1}\right) \tilde{\phi}\left(\theta_{3}-z_{2}\right) p^{\chi^{( \pm)}}(\underline{\theta}, \underline{z})\right)$. Therefore we get using (C.11) for small $\nu$ (up to constants)

$$
\begin{aligned}
K_{\overline{1} 11}^{\chi^{( \pm)}(\underline{\theta})} & =K\left(\theta_{12}, 0\right) \frac{\exp \left(\mp \frac{1}{2} \theta_{3}\right)}{\sinh \frac{1}{2} \theta_{13} \sinh \frac{1}{2} \theta_{23}}+(2 \leftrightarrow 3) \\
& =\frac{\cosh \frac{1}{2} \theta_{12}}{\left(\theta_{12}-i \pi\right) \sinh \frac{1}{2} \theta_{12}} \frac{\exp \left(\mp \frac{1}{2} \theta_{3}\right)}{\sinh \frac{1}{2} \theta_{13} \sinh \frac{1}{2} \theta_{23}}+(2 \leftrightarrow 3)+O(\nu) \\
& =\frac{1}{\theta_{12}-i \pi} \operatorname{coth} \frac{1}{2} \theta_{12} \frac{\exp \left(\mp \frac{1}{2} \theta_{3}\right)}{\sinh \frac{1}{2} \theta_{13} \sinh \frac{1}{2} \theta_{23}}+(2 \leftrightarrow 3)+O(\nu) \\
F_{\overline{1} 11}^{\chi}(\underline{\theta}) & =\frac{\cosh \frac{1}{2} \theta_{12}}{\theta_{12}-i \pi} u\left(\theta_{3}\right)-\frac{\cosh \frac{1}{2} \theta_{13}}{\theta_{13}-i \pi} u\left(\theta_{2}\right)+O(\nu)
\end{aligned}
$$

which is (E.1) up to a constant. The normalization is obtained by the form factor equation (iii)

$$
\begin{aligned}
\underset{\theta_{12}=i \pi}{\operatorname{Res}} F_{\overline{1} 11}^{\psi}(\underline{\theta}) & =2 i\left(1-a\left(\theta_{23}\right)\right) F_{1}^{\psi}\left(\theta_{3}\right) \\
& =\frac{4 \pi}{N}\left(\frac{1}{\sinh \theta_{23}}-\frac{1}{\theta_{23}}\right) u\left(\theta_{3}\right)+O\left(N^{-2}\right)
\end{aligned}
$$

where

$$
F_{\alpha \beta \gamma}^{\psi}(\underline{\theta})=\frac{i\left(\gamma\left(p_{1}+p_{2}+p_{3}\right)+m\right)}{8 m^{2} \cosh \frac{1}{2} \theta_{12} \cosh \frac{1}{2} \theta_{13} \cosh \frac{1}{2} \theta_{23}} F_{\alpha \beta \gamma}^{\psi \chi} \gamma(\underline{\theta}) .
$$


It has been used that

$$
\begin{aligned}
K(\theta, 0) & =-2 i \pi \frac{\cosh \frac{1}{2} z}{(z-i \pi) \sinh \frac{1}{2} z}+O(\nu) \\
\tilde{\phi}(\theta) & =\frac{-i \pi}{\sinh \frac{1}{2} \theta}+O(\nu) \\
F(\theta) & =-i \sinh \frac{1}{2} \theta+O(\nu) \\
a(\theta) & =1+\nu i \pi\left(\frac{1}{\sinh \theta}-\frac{1}{\theta}\right)+O\left(\nu^{2}\right) .
\end{aligned}
$$

\section{E.2 $1 / \mathrm{N}$ perturbation theory}

Introducing the auxiliary field $\sigma(x)$ the Lagrangian (1.1) may be written as

$$
\mathcal{L}^{\mathrm{GN}}=\bar{\psi}(i \gamma \partial-\sigma) \psi-\frac{1}{2 g^{2}} \sigma^{2}
$$

and the Green's functions in $1 / N$ expansions are obtained from the expansion of

$$
\begin{aligned}
& Z(\xi, \bar{\xi})=\int d \sigma \exp \left(i \mathcal{A}_{\mathrm{eff}}(\sigma)-\bar{\xi} S \xi\right) \\
& \mathcal{A}_{\mathrm{eff}}(\sigma)=-i \frac{1}{2} N \operatorname{tr} \ln (i \gamma \partial-\sigma)-\int d^{2} x \frac{1}{2 g^{2}} \sigma^{2}
\end{aligned}
$$

with the $\sigma$ propagator $[5,6]$

$$
\tilde{\Delta}_{\sigma}(k)=\left(\frac{1}{2} N \int \frac{d^{2} p}{(2 \pi)^{2}} \operatorname{tr}\left(\frac{1}{\gamma p-m}\left(\frac{1}{\gamma(p+k)-m}-\frac{1}{m}\right)\right)\right)^{-1}=-\frac{4 \pi i}{N} \frac{\tanh \frac{1}{2} \phi}{\phi}
$$

where $k^{2}=-4 m^{2} \sinh ^{2} \frac{1}{2} \phi$. This propagator together with the simple vertex of figure 5 yield the Feynman rules which allow to calculate general vertex functions in the $1 / N$ expansion. For example the four point vertex function is

$$
\tilde{\Gamma}_{A B \alpha \beta}^{(4) D C \delta \gamma}\left(-p_{3},-p_{4}, p_{1}, p_{2}\right)=\delta_{\alpha}^{\delta} \delta_{\beta}^{\gamma} G_{A B}^{D C}\left(p_{2}-p_{3}\right)-\delta_{\alpha}^{\gamma} \delta_{\beta}^{\delta} G_{A B}^{C D}\left(p_{3}-p_{1}\right)
$$

where $A, B, C, D$ are spinor indices, $\alpha \beta \gamma \delta$ are isospin indices and $G$ is given by the Feynman graph of figure 6 . Taking into account the contributions from the propagator we obtain

$$
G(k)=-1 \otimes 1 \tilde{\Delta}_{\sigma}(k)=\frac{4 \pi i}{N} 1 \otimes 1 \frac{\tanh \frac{1}{2} \phi}{\phi} .
$$

where the tensor product structure of the spinor matrices is obvious from figure 6 .

3-particle form factor of the fundamental fermi field. We now calculate the three particle form factor of the fundamental fermi field in $1 / N$-expansion in lowest nontrivial order. For convenience we multiply the field with the Dirac operator

$$
\chi^{\delta D}(x)=i(-i \gamma \partial+m)_{D^{\prime}}^{D} \psi^{\delta D^{\prime}}(x)
$$




$$
f=-
$$

Figure 5. The elementary vertex for the $O(N)$ Gross-Neveu model. With respect to isospin the vertex is proportional to the unit matrix.

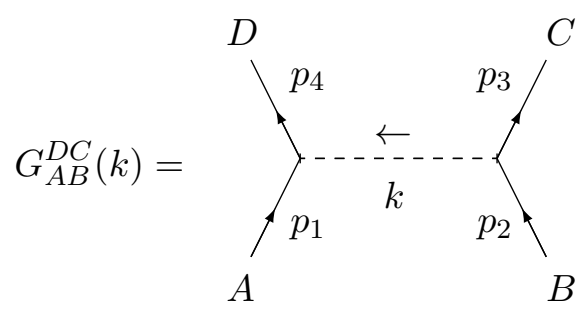

Figure 6. The four point vertex.

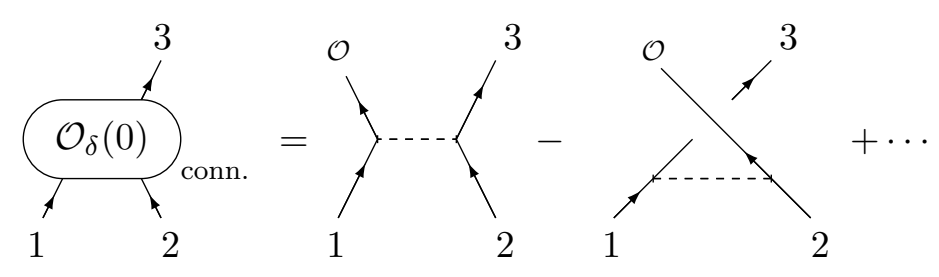

Figure 7. The connected part of the three particle form factor of the fundamental fermi field in $1 / N$-expansion.

and define

$$
{ }_{\text {out }}^{\gamma}\left\langle p_{3}\left|\chi^{\delta D}(0)\right| \theta_{1}, \theta_{2}\right\rangle_{\alpha \beta}^{\text {in }}=F_{\alpha \beta}^{\eta^{\delta D} \gamma}\left(\theta_{3} ; \theta_{1}, \theta_{2}\right)
$$

By means of LSZ-techniques one can express the connected part in terms of the 4-point vertex function (E.2) in lowest order given by the Feynman graphs of figure 7

$$
F_{\operatorname{conn} \alpha \beta}^{\chi^{\delta D} \gamma}\left(\theta_{3} ; \theta_{1}, \theta_{2}\right)=\bar{u}_{C}\left(p_{3}\right)\left\{\delta_{\alpha \delta} \delta_{\beta \gamma} G_{A B}^{D C}\left(p_{2}-p_{3}\right)-\delta_{\alpha \gamma} \delta_{\beta \delta} G_{A B}^{C D}\left(p_{3}-p_{1}\right)\right\} u^{A}\left(p_{1}\right) u^{B}\left(p_{2}\right)
$$

where $G$ is given by figure 6 and eq. (E.3) and the spinors by $u_{ \pm}(p)=\sqrt{m} e^{\mp \theta / 2}$. It turns out that for $p_{1}, p_{2}$ and $p_{3}$ on-shell several terms vanish or cancel and we obtain up to order $1 / N$ using $\bar{u}\left(\theta_{1}\right) u\left(\theta_{2}\right)=2 m \cosh \frac{1}{2} \theta_{12}$

$$
F_{\text {conn. } \alpha \beta}^{\chi^{\delta D} \gamma}=\frac{i \pi}{N} 8 m\left\{\delta_{\alpha}^{\delta} \delta_{\beta}^{\gamma} \frac{\sinh \frac{1}{2} \theta_{23}}{\theta_{23}} u^{D}\left(p_{1}\right)-\delta_{\alpha}^{\gamma} \delta_{\beta}^{\delta} \frac{\sinh \frac{1}{2} \theta_{13}}{\theta_{13}} u^{D}\left(p_{2}\right)\right\}
$$

By crossing $\left(\theta_{3} \rightarrow \theta_{3}+i \pi\right)$ this gives $F_{\alpha \beta \bar{\gamma}}^{\chi^{\delta}}$ and agrees with the $1 / N$ expansion of the exact form factor (5.11). 
Open Access. This article is distributed under the terms of the Creative Commons Attribution License (CC-BY 4.0), which permits any use, distribution and reproduction in any medium, provided the original author(s) and source are credited.

\section{References}

[1] H.M. Babujian, A. Foerster and M. Karowski, $O(N)$ matrix difference equations and a nested bethe ansatz, J. Phys. A 45 (2012) 055207.

[2] H.M. Babujian, A. Foerster and M. Karowski, The Nested Off-shell Bethe ansatz and $O(N)$ Matrix Difference Equations, arXiv:1204.3479 [INSPIRE].

[3] H.M. Babujian, A. Foerster and M. Karowski, Exact form factors of the $O(N) \sigma$-model, JHEP 11 (2013) 089 [arXiv:1308.1459] [InSPIRE].

[4] A.B. Zamolodchikov and A.B. Zamolodchikov, Relativistic Factorized S Matrix in Two-Dimensions Having O(N) Isotopic Symmetry, Nucl. Phys. B 133 (1978) 525 [InSPIRE].

[5] A.B. Zamolodchikov and A.B. Zamolodchikov, Exact S Matrix of Cross-Neveu Elementary Fermions, Phys. Lett. B 72 (1978) 481 [INSPIRE].

[6] M. Karowski and P. Weisz, Exact Form-Factors in $(1+1)$-Dimensional Field Theoretic Models with Soliton Behavior, Nucl. Phys. B 139 (1978) 455 [InSPIRE].

[7] M. Karowski and H.J. Thun, Complete S Matrix of the $O(2 N)$ Gross-Neveu Model, Nucl. Phys. B 190 (1981) 61 [inSPIRE].

[8] B. Basso, A. Sever and P. Vieira, Spacetime and Flux Tube S-Matrices at Finite Coupling for $N=4$ Supersymmetric Yang-Mills Theory, Phys. Rev. Lett. 111 (2013) 091602 [arXiv: 1303.1396] [INSPIRE].

[9] B. Basso, A. Sever and P. Vieira, Space-time S-matrix and Flux tube S-matrix II. Extracting and Matching Data, JHEP 01 (2014) 008 [arXiv:1306.2058] [INSPIRE].

[10] B. Basso, A. Sever and P. Vieira, Space-time S-matrix and Flux-tube S-matrix III. The two-particle contributions, JHEP 08 (2014) 085 [arXiv: 1402.3307] [INSPIRE].

[11] B. Basso, A. Sever and P. Vieira, Space-time S-matrix and Flux-tube S-matrix IV. Gluons and Fusion, JHEP 09 (2014) 149 [arXiv:1407.1736] [INSPIRE].

[12] S.S. Gubser, I.R. Klebanov and A.M. Polyakov, A Semiclassical limit of the gauge/string correspondence, Nucl. Phys. B 636 (2002) 99 [hep-th/0204051] [INSPIRE].

[13] D.J. Gross and A. Neveu, Dynamical Symmetry Breaking in Asymptotically Free Field Theories, Phys. Rev. D 10 (1974) 3235 [InSPIRE].

[14] R.F. Dashen, B. Hasslacher and A. Neveu, The Particle Spectrum in Model Field Theories from Semiclassical Functional Integral Techniques, Phys. Rev. D 11 (1975) 3424 [InSPIRE].

[15] B. Berg, M. Karowski and P. Weisz, Construction of Green Functions from an Exact $S$ Matrix, Phys. Rev. D 19 (1979) 2477 [InSPIRE].

[16] F. Smirnov, Form Factors in Completely Integrable Models of Quantum Field Theory, World Scientific, Adv. Ser. Math. Phys. 14 (1992).

[17] H.M. Babujian, M. Karowski and A. Zapletal, $U(N)$ matrix difference equations and a nested Bethe ansatz, J. Phys. A 30 (1997) 6425 [hep-th/9611006] [INSPIRE]. 
[18] H.M. Babujian, A. Fring, M. Karowski and A. Zapletal, Exact form-factors in integrable quantum field theories: The sine-Gordon model, Nucl. Phys. B 538 (1999) 535 [hep-th/9805185] [INSPIRE].

[19] H.M. Babujian, A. Foerster and M. Karowski, The Nested SU(N) off-shell Bethe ansatz and exact form-factors, J. Phys. A 41 (2008) 275202 [hep-th/0611012] [INSPIRE].

[20] H.M. Babujian, Off-shell Bethe Ansatz equation and $N$ point correlators in $\mathrm{SU}(2)$ WZNW theory, J. Phys. A 26 (1993) 6981 [hep-th/9307062] [inSPIRE].

[21] J.L. Cardy and G. Mussardo, Form-factors of Descendent Operators in Perturbed Conformal Field Theories, Nucl. Phys. B 340 (1990) 387 [InSPIRE].

[22] A. Fring, G. Mussardo and P. Simonetti, Form-factors for integrable Lagrangian field theories, the sinh-Gordon theory, Nucl. Phys. B 393 (1993) 413 [hep-th/9211053] [InSPIRE].

[23] V.P. Yurov and A.B. Zamolodchikov, Truncated fermionic space approach to the critical 2-D Ising model with magnetic field, Int. J. Mod. Phys. A 6 (1991) 4557 [INSPIRE].

[24] S.L. Lukyanov, Free field representation for massive integrable models, Commun. Math. Phys. 167 (1995) 183 [hep-th/9307196] [INSPIRE].

[25] S.L. Lukyanov, Form-factors of exponential fields in the sine-Gordon model, Mod. Phys. Lett. A 12 (1997) 2543 [hep-th/9703190] [INSPIRE].

[26] S.L. Lukyanov, Form-factors of exponential fields in the affine $A_{N-1}^{(1)}$ Toda model, Phys. Lett. B 408 (1997) 192 [hep-th/9704213] [INSPIRE].

[27] V. Brazhnikov and S.L. Lukyanov, Angular quantization and form-factors in massive integrable models, Nucl. Phys. B 512 (1998) 616 [hep-th/9707091] [INSPIRE].

[28] S.L. Lukyanov and A.B. Zamolodchikov, Form-factors of soliton creating operators in the sine-Gordon model, Nucl. Phys. B 607 (2001) 437 [hep-th/0102079] [InSPIRE].

[29] P. Orland, Summing Planar Diagrams by an Integrable Bootstrap, Phys. Rev. D 84 (2011) 105005 [arXiv: 1108.0058 ] [INSPIRE].

[30] B. Pozsgay, W.-V. van Gerven Oei and M. Kormos, On Form Factors in nested Bethe Ansatz systems, J. Phys. A 45 (2012) 465007 [arXiv:1204.4037] [INSPIRE].

[31] S. Pakuliak, É. Ragoucy and N.A. Slavnov, GL(3)-Based Quantum Integrable Composite Models. I. Bethe Vectors, SIGMA 11 (2015) 063 [arXiv:1501.07566] [INSPIRE].

[32] S. Pakuliak, É. Ragoucy and N.A. Slavnov, GL(3)-Based Quantum Integrable Composite Models. II. Form Factors of Local Operators, SIGMA 11 (2015) 064 [arXiv:1502.01966] [INSPIRE].

[33] S. Pakuliak, E. Ragoucy and N.A. Slavnov, Form factors of local operators in a one-dimensional two-component bose gas, J. Phys. A 48 (2015) 435001 [arXiv:1503.00546].

[34] H.M. Babujian, A. Foerster and M. Karowski, Exact kink form factors of the $O(N)$ Gross-Neveu model, to be published.

[35] H.M. Babujian, A. Foerster and M. Karowski, Exact form factors of the O(6) Gross-Neveu model, to be published.

[36] E. Ogievetsky, P. Wiegmann and N. Reshetikhin, The Principal Chiral Field in Two-Dimensions on Classical Lie Algebras: The Bethe Ansatz Solution and Factorized Theory of Scattering, Nucl. Phys. B 280 (1987) 45 [InSPIRE]. 
[37] N.Y. Reshetikhin, The Spectrum of the Transfer Matrices Connected with Kac-Moody Algebras, Lett. Math. Phys. 14 (1987) 235.

[38] N. Yu. Reshetikhin and P.B. Wiegmann, Towards the Classification of Completely Integrable Quantum Field Theories, Phys. Lett. B 189 (1987) 125 [InSPIRE].

[39] H.M. Babujian, A. Foerster and M. Karowski, The form factor program: A review and new results, the nested $\mathrm{SU}(N)$ off-shell Bethe ansatz and the 1/N expansion, Theor. Math. Phys. 155 (2008) 512 [INSPIRE].

[40] H.J. de Vega and M. Karowski, Exact Bethe Ansatz Solution of 0(2N) Symmetric Theories, Nucl. Phys. B 280 (1987) 225 [nSPIRE].

[41] M. Karowski and H.J. Thun, Complete S Matrix of the Massive Thirring Model, Nucl. Phys. B 130 (1977) 295 [INSPIRE].

[42] M. Karowski, On the Bound State Problem in $(1+1)$-dimensional Field Theories, Nucl. Phys. B 153 (1979) 244 [inSPIRE].

[43] H. Babujian and M. Karowski, Exact form-factors in integrable quantum field theories: The sine-Gordon model. 2., Nucl. Phys. B 620 (2002) 407 [hep-th/0105178] [INSPIRE].

[44] http://mathworld.wolfram.com/BarnesG-Function.html.

[45] V. Tarasov, Algebraic Bethe Ansatz for the Izergin-Korepin R Matrix, Theor. Math. Phys. 76 (1988) 793.

[46] H.M. Babujian, A. Foerster and M. Karowski, Asymptotic clustering of exact form factors and short distance behavior in integrable Quantum Field Theories, to be published. 